\title{
Chiral properties of domain-wall quarks in quenched QCD
}

\author{
A. Ali Khan, ${ }^{1}$ S. Aoki, ${ }^{2}$ Y. Aoki, ${ }^{1,2,3}$ R. Burkhalter, ${ }^{1,2}$ S. Ejiri, ${ }^{1}$ M. Fukugita, ${ }^{4}$ S. Hashimoto, ${ }^{5}$ N. Ishizuka, ${ }^{1,2}$ Y. Iwasaki, ${ }^{1,2}$ \\ T. Izubuchi, ${ }^{6}$ K. Kanaya,${ }^{1,2}$ T. Kaneko,${ }^{5}$ Y. Kuramashi,${ }^{5,7}$ T. Manke, ${ }^{1}$ K. I. Nagai, ${ }^{1}$ J. Noaki, ${ }^{1}$ M. Okawa, ${ }^{5}$ \\ H. P. Shanahan, ${ }^{1}$ Y. Taniguchi, ${ }^{2}$ A. Ukawa, ${ }^{1,2}$ and T. Yoshié ${ }^{1,2}$ \\ (CP-PACS Collaboration) \\ ${ }^{1}$ Center for Computational Physics, University of Tsukuba, Tsukuba, Ibaraki 305-8577, Japan \\ ${ }^{2}$ Institute of Physics, University of Tsukuba, Tsukuba, Ibaraki 305-8571, Japan \\ ${ }^{3}$ RIKEN BNL Research Center, Brookhaven National Laboratory, Upton, New York 11973 \\ ${ }^{4}$ Institute for Cosmic Ray Research, University of Tokyo, Tanashi, Tokyo 188-8502, Japan \\ ${ }^{5}$ High Energy Accelerator Research Organization (KEK), Tsukuba, Ibaraki 305-0801, Japan \\ ${ }^{6}$ Department of Physics, Kanazawa University, Kanazawa, Ishikawa 920-1192, Japan \\ ${ }^{7}$ Department of Physics, Washington University, St. Louis, Missouri 63130
}

(Received 6 September 2000; published 11 May 2001)

\begin{abstract}
We investigate the chiral properties of quenched domain-wall QCD (DWQCD) at the lattice spacings $a^{-1}$ $\simeq 1$ and $2 \mathrm{GeV}$ for both plaquette and renormalization-group (RG) improved gauge actions. In the case of the plaquette action we find that the quark mass defined through the axial Ward-Takahashi identity remains non-vanishing in the DWQCD chiral limit that the bare quark mass $m_{f} \rightarrow 0$ and the length of the fifth dimension $N_{s} \rightarrow \infty$, indicating that chiral symmetry is not realized with quenched DWQCD up to $a^{-1} \simeq 2 \mathrm{GeV}$. The behavior is much improved for the RG-improved gauge action: while a nonvanishing quark mass remains in the chiral limit at $a^{-1} \simeq 1 \mathrm{GeV}$, the result at $a^{-1} \simeq 2 \mathrm{GeV}$ is consistent with an exponentially vanishing quark mass in the DWQCD chiral limit, indicating the realization of exact chiral symmetry. An interpretation and implications are briefly discussed.
\end{abstract}

DOI: 10.1103/PhysRevD.63.114504

PACS number(s): 12.38.Gc, 11.15.Ha, 11.30.Rd

\section{INTRODUCTION}

A recent exciting development in lattice field theory is the realization of exact chiral-like symmetry at finite lattice spacing without fermion doubling. It started with the domainwall $[1,2]$ and the overlap [3] formalisms, which were originally proposed to formulate chiral gauge theories. They were soon applied to vector gauge theories such as QCD [3-7], and progress culminated in the recent discovery [8] that the essential feature of these formalisms is the existence of chiral-like symmetry which follows from the GinspargWilson relation [9]. An explicit form of the fermion action satisfying this relation is already known [6]. These developments lead us to expect that QCD with exact chiral symmetry can be numerically simulated on a lattice.

The domain-wall fermion formalism is a five-dimensional extension of the Wilson fermion action with a negative mass $-M$. The theoretical basis is that the effective fourdimensional theory obtained by integrating out the heavy unphysical modes satisfies the Ginsparg-Wilson relation $[10,11]$. On the practical side, the knowledge accumulated on the Wilson fermion action over the past twenty years enables an efficient implementation of this system on computers.

There are, however, two subtleties that need to be clarified for numerical applications of the domain wall formalism to QCD (DWQCD). First, the length of the fifth dimension $N_{s}$ cannot be set to infinity: one has to study the finite $N_{s}$ effect. Second, one has to tune the domain-wall height (i.e., fifth dimensional mass) $M$ to an appropriate value in order to keep the massless mode.

In the free theory one massless mode exists in the range
$0<M<2$ [4]. The two chiral modes, with opposite chiralities, are exponentially bound to the opposite boundaries in the fifth dimension. The situation remains unchanged in perturbation theory. An explicit calculation at one-loop order shows [12-14] the existence of the massless mode guaranteed for the range $0<M^{\prime}<2$ where $M^{\prime}$ receives a shift by the one-loop quantum correction. It is also easy to demonstrate that finite $N_{s}$ effects are suppressed exponentially in $N_{s}$.

Numerical tests of chiral properties and physical applications of (quenched) DWQCD were first discussed in Ref. [15]. Through measurements of pion mass and the $K^{0}-\bar{K}^{0}$ mixing matrix element at the gauge coupling $\beta=6 / \mathrm{g}^{2} \sim 6.0$ $\left(a^{-1} \approx 2 \mathrm{GeV}\right)$, these quantities were shown to vanish in the chiral limit, as required by chiral symmetry, for a rather small fifth dimensional length of $N_{s} \sim 10$. Good chiral behavior has also been observed for $N_{s} \sim 10$ for a gauge field background with non-trivial topology [16]. Even the AtiyahSinger index theorem has been shown to be approximately satisfied on a lattice for a non-zero quark mass $[16,17]$.

These successful results have promoted further applications. The calculations include the strange quark mass using nonperturbative renormalization factors [18-20], the pion decay constant [21], the hadron mass spectrum [22,23] and QCD thermodynamics with dynamical quarks [24-26].

Earlier studies with DWQCD were limited to lattice spacings of around $a^{-1} \approx 2 \mathrm{GeV}$. It is obviously necessary to investigate the scaling behavior and carry out the continuum extrapolation. Reducing the lattice spacing from $a^{-1} \simeq 2$ $\mathrm{GeV}$, however, is computationally difficult, since the formalism requires $N_{s}$ times more CPU time than usual simula- 
tions. Simulations at coarser lattices are easier, but we must clarify how large one can increase the lattice spacing while keeping the massless mode.

The chiral properties of quenched DWQCD have been examined in a number of recent reports [23,27-29] employing large fifth dimensional lengths up to $N_{s} \sim 50$. The authors of Refs. [23,27], who made a study with the plaquette gauge action at $\beta=5.7$ and $5.85\left(a^{-1} \approx 1-1.5 \mathrm{GeV}\right)$, claim that pion mass at such strong couplings does not vanish in the chiral limit after the $N_{s} \rightarrow \infty$ extrapolation, indicating that exact chiral symmetry is not realized. Our own study at $\beta$ $=5.65[29]$ yields the same conclusion. We also find that a renormalization-group (RG) improved gluon action [30] does not improve the situation at $a^{-1} \sim 1 \mathrm{GeV}$ [29].

In this paper we present a more systematic study of the chiral property of DWQCD covering both strong and "weak" coupling regions, corresponding to lattice spacings $a^{-1} \simeq 1$ and $2 \mathrm{GeV}$ with both plaquette and RG improved actions. We emphasize that the chiral properties are best studied with the quark mass extracted by the WardTakahashi (WT) identity for the axial vector current. The difference of this mass and the bare quark mass defines what we call "anomalous quark mass." We discuss that this quantity suffers from much less systematic uncertainties than the pion mass from both theoretical and numerical points of view. We study the behaviors of the anomalous quark mass as a function of $N_{s}$ and examine whether it vanishes exponentially in the $N_{s} \rightarrow \infty$ limit for various coupling regimes, as required by the self-consistency of the proper chiral theory. This study is also made with varying $M$ and a fourdimensional spatial extent at some fixed coupling. We carry out this study with a parallel set of simulations employing both plaquette and RG-improved gauge actions to see the effect of improvement of the gauge field dynamics on the chiral property.

In the case of the plaquette action we find that the anomalous quark mass remains non-vanishing in the limit that $m_{f}$ $\rightarrow 0$ and $N_{s} \rightarrow \infty$, indicating that chiral symmetry is not realized up to $a^{-1} \simeq 2 \mathrm{GeV}$. On the other hand, the behavior is much improved for the RG-improved gauge action: while a non-vanishing quark mass remains at $a^{-1} \simeq 1 \mathrm{GeV}$, the anomalous quark mass seems to vanish exponentially in $N_{s}$ at $a^{-1} \simeq 2 \mathrm{GeV}$, suggesting the realization of exact chiral symmetry. In connection to our work we refer to Ref. [28], which attempts to extract the WT identity quark mass from the pseudoscalar susceptibility.

This paper is organized as follows. In Sec. II we define the action and the anomalous quark mass. Numerical simulations and run parameters are described in Sec. III. In Sec. IV we investigate the chiral property for the plaquette action, and in Sec. V for the RG-improved gauge action. Our provisional interpretation is given in Sec. VI for the results we found from the simulation. We close the paper with a brief summary and comments on the application of DWQCD (Sec. VII). Appendix A presents an analysis with pion mass, and discuss whether they support the conclusions based on the anomalous quark mass. Numerical data for the anomalous quark mass and pion mass are collected in Appendix B.

\section{ACTION AND AXIAL WARD-TAKAHASHI IDENTITY}

We adopt Shamir's domain-wall fermion action [4,5], except that the Wilson term and the domain wall height $M$ have minus signs:

$$
\begin{aligned}
S_{f}= & -\sum_{x, s, y, s^{\prime}} \bar{\psi}(x, s) D_{d w f}\left(x, s ; y, s^{\prime}\right) \psi\left(y, s^{\prime}\right) \\
& +\sum_{x} m_{f} \bar{q}(x) q(x), \\
D_{d w f}\left(x, s ; y, s^{\prime}\right) & =D^{4}(x, y) \delta_{s, s^{\prime}}+D^{5}\left(s, s^{\prime}\right) \delta_{x, y} \\
& +(M-5) \delta_{x, y} \delta_{s, s^{\prime}}, \\
D^{4}(x, y)= & \sum_{\mu} \frac{1}{2}\left[\left(1-\gamma_{\mu}\right) U_{x, \mu} \delta_{x+\hat{\mu}, y}\right. \\
& \left.+\left(1+\gamma_{\mu}\right) U_{y, \mu}^{\dagger} \delta_{x-\hat{\mu}, y}\right], \\
D^{5}\left(s, s^{\prime}\right)= & \begin{cases}P_{L} \delta_{2, s^{\prime}} & (s=1), \\
P_{L} \delta_{s+1, s^{\prime}}+P_{R} \delta_{s-1, s^{\prime}} & \left(1<s<N_{s}\right), \\
P_{R} \delta_{N_{s}-1, s^{\prime}} & \left(s=N_{s}\right),\end{cases}
\end{aligned}
$$

where $x, y$ are four-dimensional space-time coordinates, and $s, s^{\prime}$ are fifth-dimensional or "flavor" indexes, bounded as $1 \leqslant s, s^{\prime} \leqslant N_{s}$ with the free boundary condition at both ends (we assume $N_{s}$ to be even); $P_{R / L}$ is the projection matrix $P_{R / L}=\left(1 \pm \gamma_{5}\right) / 2$, and $m_{f}$ is physical quark mass.

In DWQCD the zero mode of a domain-wall fermion is extracted by the "physical" quark field defined on the edges of the fifth dimensional space:

$$
\begin{aligned}
& q(x)=P_{L} \psi(x, 1)+P_{R} \psi\left(x, N_{s}\right), \\
& \bar{q}(x)=\bar{\psi}\left(x, N_{s}\right) P_{L}+\bar{\psi}(x, 1) P_{R} .
\end{aligned}
$$

The QCD operators are constructed from these quark fields.

For the gauge part of the action we employ the following form in 4 dimensions:

$$
\begin{aligned}
S_{\text {gluon }}= & \frac{1}{g^{2}}\left\{c_{0} \sum_{\text {plaquette }} \operatorname{Tr} U_{p l}+c_{1} \sum_{\text {rectangle }} \operatorname{Tr} U_{r t g}\right. \\
& \left.+c_{2} \sum_{\text {chair }} \operatorname{Tr} U_{\text {chr }}+c_{3} \sum_{\text {parallelogram }} \operatorname{Tr} U_{p l g}\right\},
\end{aligned}
$$

where the first term represents the standard plaquette action, and the remaining terms are six-link loops formed by a 1 $\times 2$ rectangle, a bent $1 \times 2$ rectangle (chair) and a 3 -dimensional parallelogram. The coefficients $c_{0}, \ldots, c_{3}$ satisfy the normalization condition

$$
c_{0}+8 c_{1}+16 c_{2}+8 c_{3}=1 .
$$


The standard Wilson plaquette action is given by $c_{0}=1, c_{1}$ $=c_{2}=c_{3}=0$. The RG-improved action of Iwasaki [30] is defined by setting the parameters to $c_{0}=3.648, c_{1}$ $=-0.331, c_{2}=c_{3}=0$ : with this choice of parameters the action is expected to realize smooth gauge field fluctuations approximating those in the continuum limit better than with the unimproved plaquette action.

The chiral transformation of domain wall fermions is defined as [5]

$$
\begin{aligned}
& \delta \psi(x, s)=i Q(s) \epsilon^{a} T^{a} \psi(x, s), \\
& \delta \bar{\psi}(x, s)=-i \bar{\psi}(x, s) Q(s) \epsilon^{a} T^{a},
\end{aligned}
$$

where $Q(s)=\operatorname{sgn}\left(N_{s}-2 s+1\right), T^{a}$ is a generator of flavor symmetry group $\mathrm{SU}\left(N_{f}\right)$ with $\epsilon^{a}$ a transformation parameter. The corresponding WT identity becomes

$$
\sum_{\mu}\left\langle\nabla_{\mu} A_{\mu}^{a}(x) \mathcal{O}\right\rangle=2 m_{f}\left\langle P^{a}(x) \mathcal{O}\right\rangle+2\left\langle J_{5 q}^{a}(x) \mathcal{O}\right\rangle+i\left\langle\delta_{x}^{a} \mathcal{O}\right\rangle,
$$

where the axial-vector current $A_{\mu}^{a}(x)$ and the pseudoscalar density $P^{a}(x)$ are defined by

$$
\begin{aligned}
A_{\mu}^{a}(x)= & \sum_{s} Q(s) \frac{1}{2}\left(\bar{\psi}(x, s) T^{a}\left(1-\gamma_{\mu}\right) U_{\mu}(x) \psi(x+\mu, s)\right. \\
& \left.-\bar{\psi}(x+\mu, s)\left(1+\gamma_{\mu}\right) U_{\mu}^{\dagger}(x) T^{a} \psi(x, s)\right), \\
P^{a}(x)= & \bar{q}(x) T^{a} \gamma_{5} q(x),
\end{aligned}
$$

and $J_{5 q}^{a}(x)$ representing explicit breaking of chiral symmetry takes the form

$$
\begin{aligned}
J_{5 q}^{a}(x)= & -\bar{\psi}\left(x, \frac{N_{s}}{2}\right) T^{a} P_{L} \psi\left(x, \frac{N_{s}}{2}+1\right) \\
& +\bar{\psi}\left(x, \frac{N_{s}}{2}+1\right) T^{a} P_{R} \psi\left(x, \frac{N_{s}}{2}\right) .
\end{aligned}
$$

For a smooth gauge field background the anomalous contribution $\left\langle J_{5 q}^{a}(x) \mathcal{O}^{b}(y)\right\rangle$ is bounded by an exponentially small value with the argument $-N_{s}$ and vanishes in the limit $N_{s} \rightarrow \infty$ [5]. In this paper we adopt the operator $\mathcal{O}$ $=P^{b}(0, \mathbf{0})$, and measure the chiral symmetry breaking effect by

$$
m_{5 q}=\lim _{t \rightarrow \infty} \frac{\sum_{\mathbf{x}}\left\langle J_{5 q}^{a}(t, \mathbf{x}) P^{b}(0, \mathbf{0})\right\rangle}{\sum_{\mathbf{x}}\left\langle P^{a}(t, \mathbf{x}) P^{b}(0, \mathbf{0})\right\rangle},
$$

which we call "anomalous quark mass." We expect this quantity to be dominated by short-range fluctuations since it is determined by the coupling strength of pion to the operators $J_{5 q}^{a}$ and $P^{b}$. Therefore, finite-size effects are likely to be small. For the same reason, there should be no quenched chiral singularities in the relation between $m_{5 q}$ and the bare quark mass $m_{f}$.

The axial WT identity is written in terms of the anomalous quark mass as

$$
\frac{\sum_{\mathbf{x}} \nabla_{\mu}\left\langle A_{\mu}^{a}(t, \mathbf{x}) P^{b}(0, \mathbf{0}\rangle\right)}{2 \sum_{\mathbf{x}}\left\langle P^{a}(t, \mathbf{x}) P^{b}(0, \mathbf{0})\right\rangle}=m_{f}+m_{5 q},
$$

at large $t$. The left-hand side of this equation, proportional to the pion mass squared, vanishes at $m_{f}+m_{5 q}=0$.

\section{RUN PARAMETERS AND MEASUREMENTS}

We explore the chiral properties of DWQCD at both $a^{-1} \simeq 1$ and $2 \mathrm{GeV}$ using the plaquette and the RG-improved actions. For the plaquette action, we choose the couplings $\beta=5.65$ and 6.0 , which correspond to $a^{-1}=1.00$ and 2.00 $\mathrm{GeV}$, respectively, if one determines the scale from the string tension $\sigma$ [31] assuming $\sigma=(440 \mathrm{MeV})^{2}$. For the RGimproved action, we take $\beta=2.2$ and 2.6 for which $a^{-1}$ $=0.97$ and $1.94 \mathrm{GeV}$ also from the string tension reported in Refs. [32-34].

We generate quenched gauge configurations on fourdimensional $N_{\sigma}^{3} \times N_{\tau}$ lattices. One sweep of the gauge update contains one pseudo-heatbath and four over-relaxation steps. For runs at $a^{-1} \simeq 1 \mathrm{GeV}$ we adopt the lattice size of $12^{3}$ $\times 24$ and $16^{3} \times 24$ to study finite-size effects. At $a^{-1} \simeq 2$ $\mathrm{GeV}$ only a $16^{3} \times 32$ lattice is employed. After a thermalization of 500 (2000) sweeps hadron propagators are calculated at every 100th (200th) sweeps for $a^{-1} \simeq 1 \mathrm{GeV}(2 \mathrm{GeV})$.

The domain-wall quark propagator is calculated on $N_{\sigma}^{3}$ $\times N_{\tau} \times N_{s}$ lattices, where the gauge configuration on each fifth dimensional coordinate $s$ is identical and is fixed to the Coulomb gauge. The size of the fifth dimension is varied from $N_{s}=10$ to 50 at $a^{-1} \simeq 1 \mathrm{GeV}$, and from $N_{s}=4$ to 24-30 at $a^{-1} \simeq 2 \mathrm{GeV}$, depending on the gauge action.

To allow a chiral extrapolation, we employ fermion masses of $m_{f}=0.03,0.05,0.1$ (at $a^{-1} \simeq 1 \mathrm{GeV}$ ) and $m_{f}$ $=0.02,0.04,0.06$ (at $\left.a^{-1} \simeq 2 \mathrm{GeV}\right)$. The domain-wall height dependence is examined at $a^{-1} \simeq 1 \mathrm{GeV}$ with the choice of $M=1.3,1.7,2.1$, and 2.5. At $a^{-1} \simeq 2 \mathrm{GeV}$ we choose $M$ $=1.8$.

The propagators are calculated with the conjugate gradient algorithm with an even-odd pre-conditioning. We place the source at $s=1$ and $N_{s}$ in the fifth coordinate, so that the propagator from the physical field $\bar{q}$ to the domain-wall field $\psi_{x, s}$ for arbitrary $(x, s)$ is obtained. Both local and exponentially smeared sources are employed in the spatial directions, and meson masses are measured for all possible combinations of quark masses while only degenerate combinations are evaluated for anomalous quark masses.

Our simulation parameters and the number of configurations are given in Table I. We note that gauge configurations are generated independently for each choice of $N_{s}$ and $M$, i.e., there are no correlations between the data generated with 
TABLE I. Number of configurations for the plaquette action and the RG improved action (RG) for each $N_{s}$. The anomalous quark mass is measured only at the points where number of configurations is written in boldface.

\begin{tabular}{|c|c|c|c|c|c|c|c|c|c|c|}
\hline \multirow[b]{2}{*}{$N_{s}$} & \multicolumn{5}{|c|}{ Plaquette $(\beta=5.65)$} & \multicolumn{5}{|c|}{$\mathrm{RG}(\beta=2.2)$} \\
\hline & 10 & 20 & 30 & 40 & 50 & 10 & 20 & 30 & 40 & 50 \\
\hline$M$ & \multicolumn{10}{|c|}{$12^{3} \times 24$} \\
\hline 1.3 & 20 & 20 & 20 & 20 & 20 & 20 & 20 & 20 & 20 & 20 \\
\hline 1.7 & 30 & 20 & 20 & 20 & 20 & 30 & 30 & 20 & 40 & 20 \\
\hline 2.1 & 30 & 30 & 30 & 20 & 20 & 30 & 30 & 20 & 20 & 20 \\
\hline 2.5 & 30 & 20 & 20 & - & 10 & 30 & 30 & 20 & - & - \\
\hline$M$ & \multicolumn{10}{|c|}{$16^{3} \times 24$} \\
\hline 1.3 & 20 & 20 & - & - & 20 & 20 & - & 24 & - & 24 \\
\hline 1.7 & 20 & 20 & 20 & 20 & 20 & 20 & 24 & 20 & 20 & 24 \\
\hline 2.1 & 20 & 20 & 20 & 20 & 20 & 20 & 20 & 20 & 20 & 24 \\
\hline & \multicolumn{5}{|c|}{ Plaquette $(\beta=6.00)$} & \multicolumn{5}{|c|}{$\mathrm{RG}(\beta=2.6)$} \\
\hline$N_{s}$ & 4 & 10 & 16 & 20 & 30 & 4 & 10 & 16 & 20 & 24 \\
\hline$M$ & \multicolumn{10}{|c|}{$16^{3} \times 32$} \\
\hline 1.8 & 20 & 40 & 60 & 80 & 100 & 20 & 40 & 60 & 80 & 100 \\
\hline
\end{tabular}

a different domain-wall height and a fifth-dimensional size.

In Figs. 1 and 2 we show typical data for the ratio of two-point functions defined in Eq. (2.14) as a function of the temporal distance $t$. We obtain the anomalous quark mass $m_{5 q}$ at each $m_{f}, M$ and $N_{s}$, by fitting the plateau with a constant, the fitting range being determined by the inspection of plots for the ratio and those for the effective pion mass. We choose $8 \leqslant t \leqslant 16$ as the fitting range for all simulations at $\beta=5.65$ (plaquette) and $\beta=2.2(\mathrm{RG})$. We use $10 \leqslant t \leqslant 22$ at $\beta=6.0$ for the plaquette action and $\beta=2.6$ for the RG action. The numerical values for $m_{5 q}$ and pion mass are given in Appendix B.

\section{PLAQUETTE GAUGE ACTION}

We discuss the anomalous quark mass for the plaquette gauge action. The bare quark mass $\left(m_{f}\right)$ dependence of the anomalous quark mass $m_{5 q}$ is illustrated in Fig. 3 for $\beta$ $=5.65$ and 6.0. We observe only a mild dependence of $m_{5 q}$ on $m_{f}$. The anomalous quark mass in the nominal chiral limit $m_{5 q}\left(m_{f}=0, N_{s}, M\right)$ is obtained by a linear extrapolation in $m_{f}$. The extrapolated values are collected in Appendix B.

If chiral symmetry is realized in the $N_{s} \rightarrow \infty$ limit of the DWQCD system, $m_{5 q}\left(m_{f}=0, N_{s}, M\right)$ should vanish exponentially in $N_{s}$. We examine this point first at a strong coupling $\beta=5.65$ corresponding to $a^{-1} \simeq 1 \mathrm{GeV}$. The anomalous quark mass in the nominal chiral limit, $m_{5 q}\left(m_{f}\right.$ $\left.=0, N_{s}, M\right)$, is plotted as a function of $N_{s}$ in Fig. 4 for three values of $M(M=1.3,1.7,2.1)$. A comparison of the filled symbols taken on a $16^{3} \times 24 \times N_{s}$ lattice with the open ones on a spatially smaller $12^{3} \times 24 \times N_{s}$ lattice shows no significant finite-size effect between the two spatial sizes corresponding to $N_{\sigma} a \simeq 2.4$ and $3.2 \mathrm{fm}$. (a) plaquette, $\beta=5.65,16^{3} \times 24 \times 30, M=1.7$

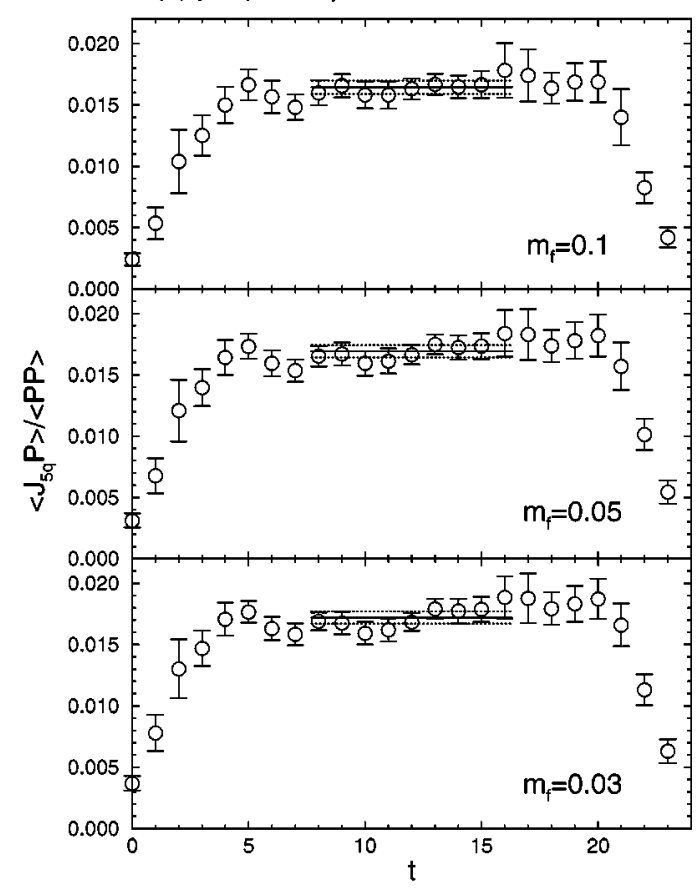

(b) plaquette, $\beta=6.0,16^{\circ} 32 \times 30, M=1.8$

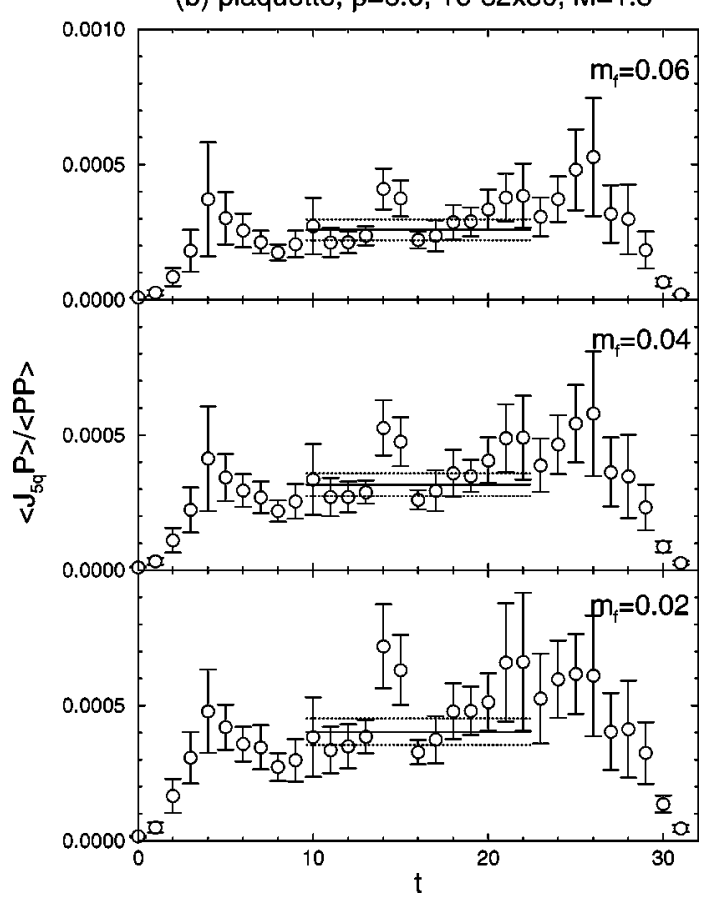

FIG. 1. Ratio of two point functions $\left\langle J_{5 q}(t) P(0)\right\rangle /\langle P(t) P(0)\rangle$ for plaquette action at $\beta=5.65, M=1.7$ on a $16^{3} \times 24 \times 30$ lattice (a) and at $\beta=6.0, M=1.8$ on a $16^{3} \times 32 \times 30(\mathrm{~b})$. Lines show constant fit over the fitted range.

The solid lines in Fig. 4 show the results of fitting data taken on a $16^{3} \times 24 \times N_{s}$ lattice to an exponential with a constant, $c+\alpha e^{-\xi N_{s}}$. This fit reproduces all five data points with an acceptable $\chi^{2} / \mathrm{DOF}$ for both $M=1.7\left(\chi^{2} / \mathrm{DOF}=1.1\right)$ and $M=2.1\left(\chi^{2} / \mathrm{DOF}=2.2\right)$. In contrast an exponential fit without a constant, $\alpha e^{-\xi N_{s}}$, does not work, resulting in a large $\chi^{2} / \mathrm{DOF}>30-60$ (dotted lines). 
(a) RG improved, $\beta=2.2,16^{3} \times 24 \times 30, M=1.7$

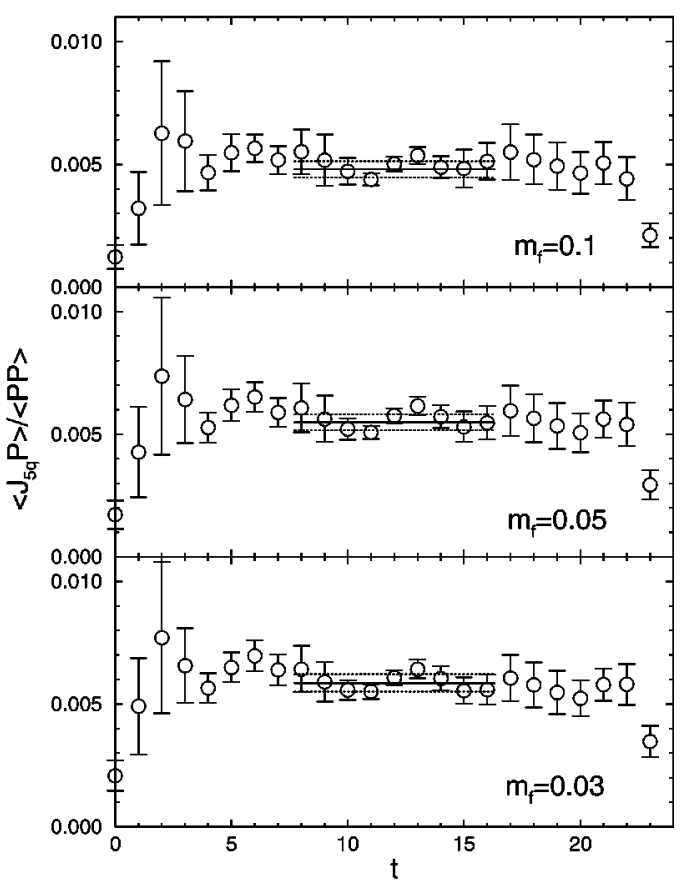

(b) $R G$ improved, $\beta=2.6,16^{\circ} \times 32 \times 24, M=1.8$

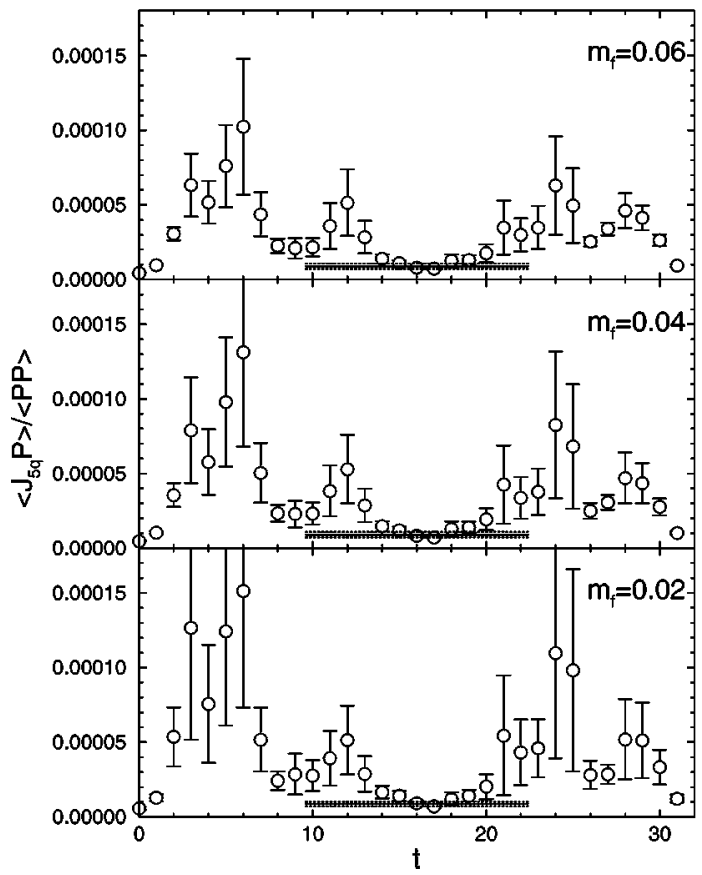

FIG. 2. Ratio of two point functions $\left\langle J_{5 q}(t) P(0)\right\rangle /\langle P(t) P(0)\rangle$ for RG improved action at $\beta=2.2, M=1.7$ on a $16^{3} \times 24 \times 30$ lattice (a) and at $\beta=2.6, M=1.8$ on a $16^{3} \times 32 \times 24$ lattice (b). Lines show constant fit over the fitted range.

In order to examine the stability of the fit, we carry out fits to the four points excluding the data for the smallest fifth dimensional length $N_{s}=10$. As seen in Table II, the data are again fitted well with an exponential plus a constant, but not without a constant. The constant $c$ agrees between the fiveand four-point fits within statistical errors.

Our data at $\beta=6.0$ are shown in Fig. 5 where, for com-
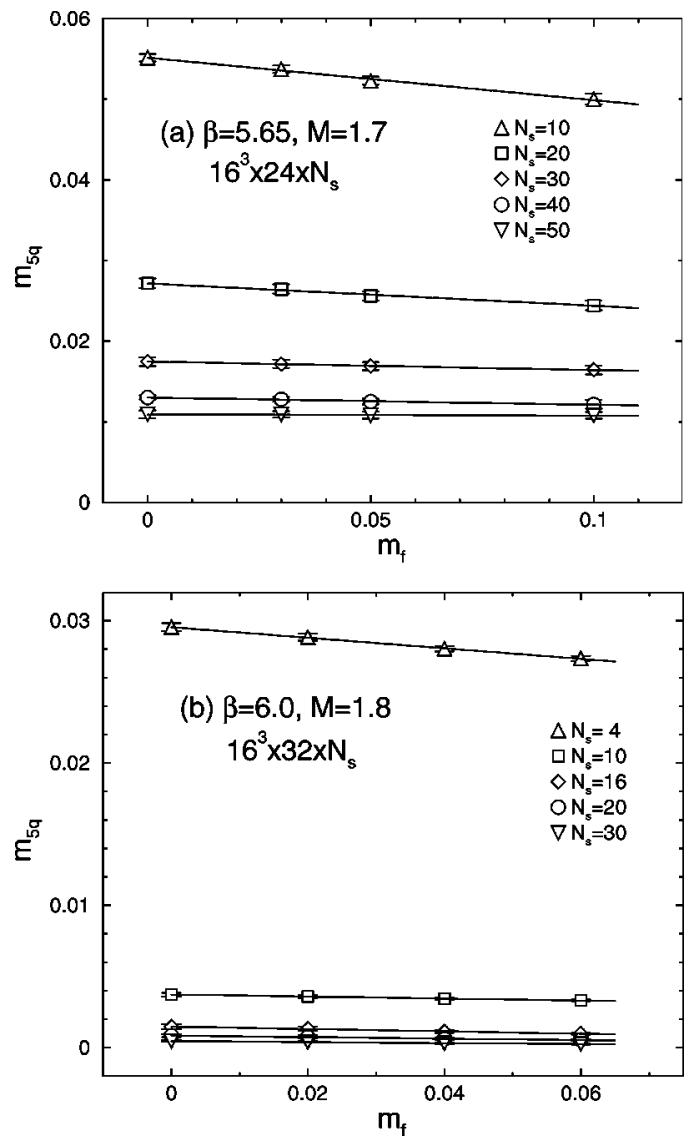

FIG. 3. Anomalous quark mass $m_{5 q}$ as a function of $m_{f}$ for the plaquette action at $M=1.7$ on a $16^{3} \times 24 \times N_{s}$ lattice in the strong coupling region (a) and at $M=1.8$ on a $16^{3} \times 32 \times N_{s}$ lattice in the weak coupling region (b).

parison, the result at $\beta=5.65$ and $M=1.7$ are recapitulated from Fig. 4. One generally expects that the domain-wall formulation works better at weaker couplings [5]. Indeed, the decrease of $m_{5 q}$ as a function of $N_{s}$ is much more rapid at

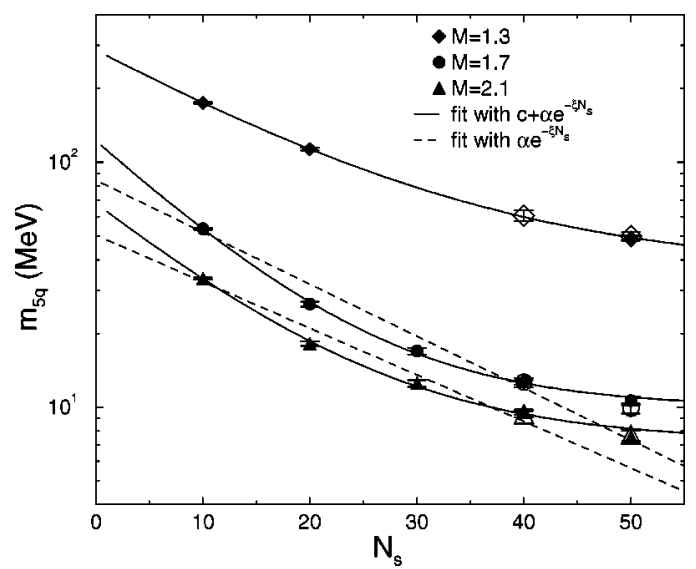

FIG. 4. Anomalous quark mass $m_{5 q}$ as a function of $N_{s}$ in $m_{f}$ $\rightarrow 0$ limit for the plaquette action at $\beta=5.65$. Filled symbols are data on $16^{3} \times 24 \times N_{s}$ lattice, and open ones on $12^{3} \times 24 \times N_{s}$ lattice. Lines are fits to all the filled points with two functions: $\alpha e^{-\xi N_{s}}$ (dotted line) and $c+\alpha e^{-\xi N_{s}}$ (solid line). 
TABLE II. Exponential fit of the anomalous quark mass $m_{5 q}$ for the plaquette action. Strong coupling results at $\beta=5.65$ are for the lattice size $16^{3} \times 24$, and those at a weak coupling of $\beta=6.0$ are for $16^{3} \times 32$ lattice. Fits with all of the five data points are represented by the fitting range $10-50$ or $4-30$. Four-points fits without the data of smallest $N_{s}$ are represented by $20-50$ or $10-30$.

\begin{tabular}{|c|c|c|c|c|c|c|c|c|c|}
\hline$\beta$ & $M$ & $\begin{array}{c}\text { Fitting range } \\
\text { of } N_{s}\end{array}$ & $c$ & $\begin{array}{l}c+\alpha e \\
\alpha\end{array}$ & $\xi$ & $\chi^{2} / \mathrm{DOF}$ & $\alpha$ & $\begin{array}{c}\alpha e^{-\xi N_{s}} \\
\xi\end{array}$ & $\chi^{2} / \mathrm{DOF}$ \\
\hline \multirow{4}{*}{5.65} & \multirow{3}{*}{1.7} & $10-50$ & $0.01032(46)$ & $0.1154(46)$ & $0.0947(44)$ & 1.1 & $0.0868(91)$ & $0.0488(52)$ & 59.1 \\
\hline & & $20-50$ & $0.0092(11)$ & $0.084(17)$ & $0.077(12)$ & 0.002 & $0.0514(70)$ & $0.0337(41)$ & 9.0 \\
\hline & & $10-50$ & $0.00725(63)$ & $0.0605(35)$ & $0.0838(69)$ & 2.2 & $0.0503(44)$ & $0.0438(46)$ & 34.8 \\
\hline & 2.1 & $20-50$ & $0.0052(15)$ & $0.0395(62)$ & $0.056(13)$ & 0.2 & $0.0335(22)$ & $0.0313(21)$ & 2.3 \\
\hline & 1.8 & $4-30$ & $0.00057(15)$ & $0.124(12)$ & $0.364(22)$ & 8.7 & $0.112(21)$ & $0.333(38)$ & 47.8 \\
\hline 6.0 & 1.8 & $10-30$ & $0.000396(79)$ & $0.0234(58)$ & $0.195(25)$ & 0.2 & $0.0124(30)$ & $0.123(19)$ & 7.3 \\
\hline
\end{tabular}

$\beta=6.0$, giving $m_{5 q}$ an order of magnitude smaller at $\beta$ $=6.0$ than at $\beta=5.65$ :

$$
m_{5 q}= \begin{cases}10.32(46) \mathrm{MeV} & \text { at } M=1.7, \beta=5.65 \\ 0.79(16) \mathrm{MeV} & \text { at } M=1.8, \beta=6.0\end{cases}
$$

Nonetheless, we still observe a clear flattening of $m_{5 q}$ toward a large $N_{s}$. A plain exponential fit without a residual constant does not work. This is true even if we drop the $N_{s}=4$ data. The fit including a constant is acceptable as shown by the solid lines in Fig. 5 (see Table II for details of $\chi^{2}$ analyses).

As a further test for the presence of a non-zero constant, we extract the decay rate $\xi$ in $N_{s}$ not only at the chiral limit $\left(m_{f}=0\right)$ but also at a finite $m_{f}$. According to the transfer matrix description of DWQCD $[5,10,11]$, the mass term, which appears only at the boundary in the fifth dimension, does not affect the large $N_{s}$ dependence of $m_{5 q}$; hence we expect that $\xi$ does not depend on $m_{f}$. The decay rates $\xi$ from a fit with an exponential and a constant are plotted in Fig. 6,

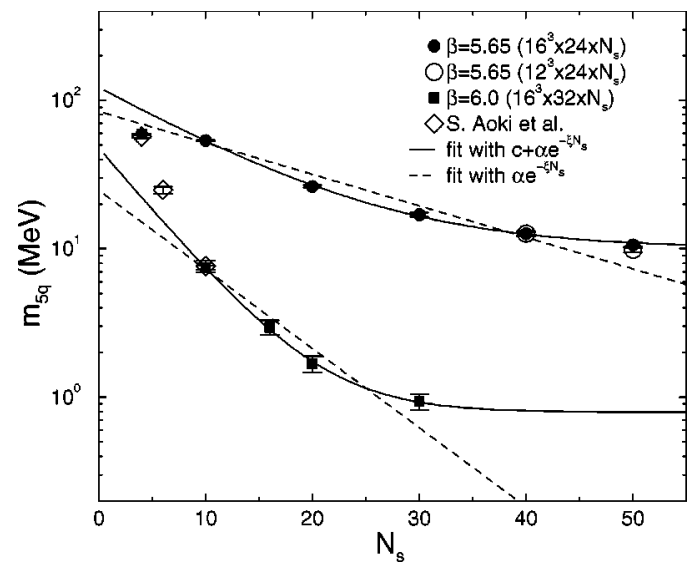

FIG. 5. Anomalous quark mass $m_{5 q}$ as a function of $N_{s}$ in $m_{f}$ $\rightarrow 0$ limit for the plaquette action at weak coupling $\beta=6.0$ with $M=1.8$ (filled squares) on a $16^{3} \times 32 \times N_{s}$ lattice. Representative strong coupling results taken at $\beta=5.65$ and $M=1.7$ are reproduced from Fig. 4 for comparison (filled and open circles). The results at $\beta=6.0$ from a previous simulation [21] is also given with open diamonds. Lines are fits to filled symbols with two functions: $\alpha e^{-\xi N_{s}}$ (dotted line) and $c+\alpha e^{-\xi N_{s}}$ (solid line). which show that this expectation is well satisfied at a strong coupling, and within estimated errors also at a weaker coupling. The decay rate extrapolated to the chiral limit from finite $m_{f}$ is consistent with the value directly extracted for $m_{f}=0$, showing the consistency of the analysis.

We conclude that a residual constant remains in the anomalous quark mass in the $N_{s} \rightarrow \infty$ limit. The presence of a residual constant, even if it is small, means that quenched DWQCD does not realize the expected chiral symmetry for the plaquette action, at least, up to $a^{-1} \simeq 2 \mathrm{GeV}$.

We remark that our conclusion differs from Ref. [21], which indicates that $m_{5 q}$ vanishes exponentially in $N_{s}$ at $\beta$ $=6.0$ and $M=1.819$ with the plaquette action on the same four-dimensional lattice size. We find that the two data for $m_{5 q}$ are mutually consistent in the overlapping range of $N_{s}$ (see Fig. 5, where open points are from Ref. [21] and the solid points denote our data). The choice of $N_{s} \leqslant 10$ in Ref. [21] was too small to observe the asymptotic flattening.

\section{RENORMALIZATION GROUP IMPROVED ACTION}

We may ascribe the failure in satisfying chiral symmetry seen in the last section to the roughness of gauge configura-

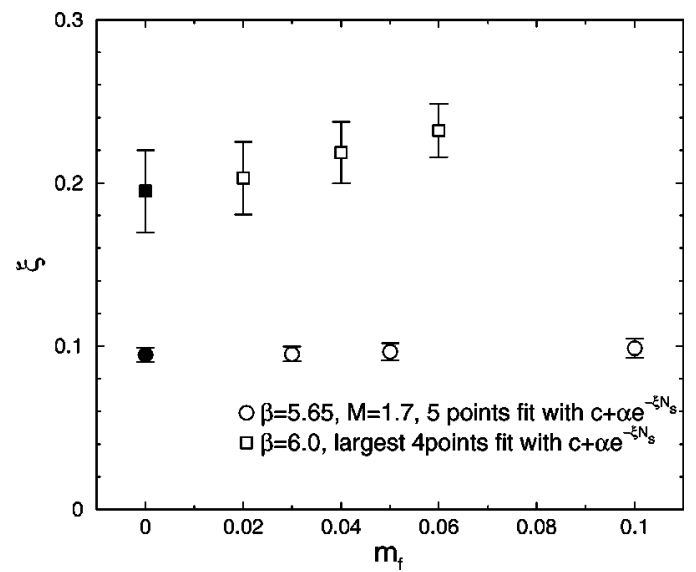

FIG. 6. Decay rate $\xi$ of the anomalous quark mass from the fit with $c+\alpha e^{-\xi N_{s}}$ for plaquette action. Open circles represent the data in the strong coupling region at $M=1.7, \beta=5.65$ on $16^{3} \times 24 \times N_{s}$ lattice with five points fit. Open squares are those in the weak coupling region at $M=1.8, \beta=6.0$ on $16^{3} \times 32 \times N_{s}$ lattice with four points fit. 


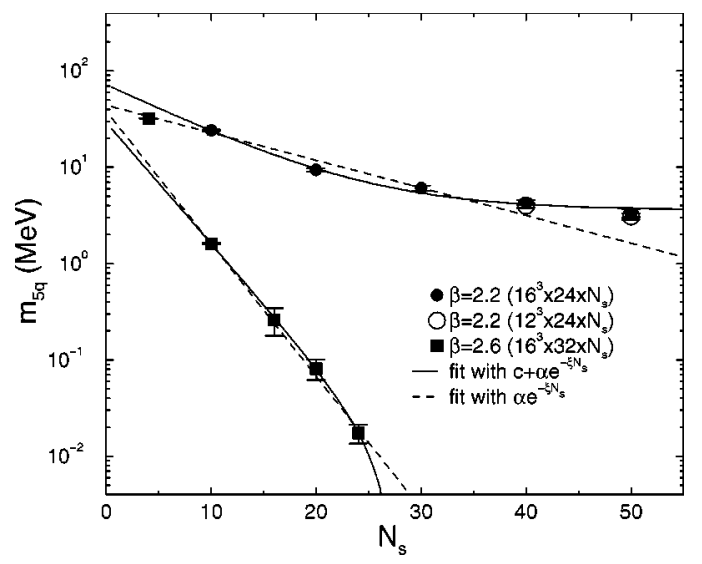

FIG. 7. Anomalous quark mass $m_{5 q}$ as a function of $N_{s}$ in $m_{f}$ $\rightarrow 0$ limit for the RG improved action. Filled circles represent data in the strong coupling region at $M=1.7$ and $\beta=2.2$ on $16^{3} \times 24$ $\times N_{s}$ lattice. Filled squares are those in the weak coupling region at $M=1.8$ and $\beta=2.6$ on $16^{3} \times 32 \times N_{s}$ lattice. Lines are fits with two functions: $\alpha e^{-\xi N_{s}}$ (dotted line) and $c+\alpha e^{-\xi N_{s}}$ (solid line). Data for $N_{s}=10-50$ (5 points) and 10-24 (4 points) are used for the fit in the strong and weak coupling.

tions with the plaquette action at the lattice spacings we have studied. We suspect that the massless mode may exist on sufficiently smooth gauge configurations close to the continuum limit $[35,36]$. One way to realize smooth gauge configurations, yet keeping the lattice spacing coarse enough to make a computation feasible, is to employ improved gauge actions. So we discuss the case with the RG-improved gauge action [30] in this section.

The anomalous quark mass in the nominal chiral limit $m_{5 q}\left(m_{f}=0, N_{s}, M\right)$ is plotted as a function of $N_{s}$ in Figs. 7 and 8 . Figure 7 compares a typical result at a strong coupling $(\beta=2.2)$ with that at a weaker coupling $(\beta=2.6)$. Figure 8 displays the results at $\beta=2.2$ for three different choices of $M$ and for two different choices of the spatial lattice size $N_{\sigma}$. A comparison of solid points $\left(16^{3} \times 24 \times N_{s}\right.$ lattices $)$ and open points $\left(12^{3} \times 24 \times N_{s}\right)$ verifies the absence of a significant finite size effect at this $\beta$. In order to take the $N_{s} \rightarrow \infty$ limit we carry out exponential fits, with or without a constant, as we did for the plaquette action. The fit parameters are summarized in Table III.

We first discuss the results in the weak coupling region

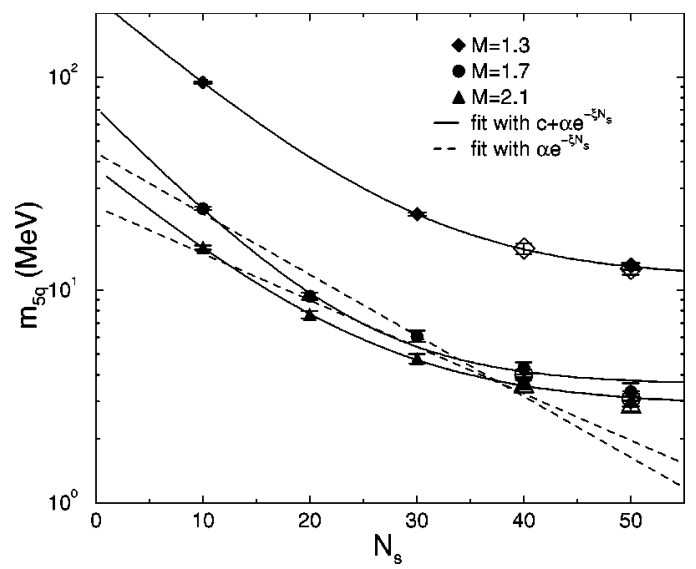

FIG. 8. Anomalous quark mass $m_{5 q}$ as a function of $N_{s}$ in $m_{f}$ $\rightarrow 0$ limit for the RG-improved action at $\beta=2.2$. Filled symbols are data on $16^{3} \times 24 \times N_{s}$ lattice, and open ones on $12^{3} \times 24 \times N_{s}$ lattice. Lines are fits to all the filled points with two functions: $\alpha e^{-\xi N_{s}}$ (dotted line) and $c+\alpha e^{-\xi N_{s}}$ (solid line).

displayed in Fig. 7. As one can see from large values of $\chi^{2} / \mathrm{DOF}$ in Table III, neither $c+\alpha e^{-\xi N_{s}}\left(\chi^{2} / \mathrm{DOF}=12.8\right)$ nor $\alpha e^{-\xi N_{s}}\left(\chi^{2} / \mathrm{DOF}=16.9\right)$ fits the data well in so far as the point at $N_{s}=4$ is included. This means that the leading exponential term does not dominate the two point correlators owing to a non-trivial overlap of quark wave functions between the two domain walls for this small $N_{s}$.

If the data at $N_{s}=4$ are excluded, however, the four points for $N_{s} \geqslant 10$ are fitted equally well with either $c$ $+\alpha e^{-\xi N_{s}}$ (solid line) or $\alpha e^{-\xi N_{s}}$ (dotted line) as seen in the figure. In fact, the fit with a constant yields $c=-0.71$ (63) $\times 10^{-5}$, consistent with zero at one standard deviation; the four data points fall on $\alpha e^{-\xi N_{s}}$, indicating the realization of chiral symmetry.

We obtain a different result for a strong coupling. Solid lines in Fig. 8 show fits to all five data points with an exponential plus a constant. They provide reasonable fits to all data. On the other hand, a plain exponential does not fit the data.

Repeating the analysis for the four data points excluding $N_{s}=10$, we still find the fit with a non-zero constant $\left(\chi^{2} / \mathrm{DOF}=0.1\right)$ being much better than a plain exponential $\left(\chi^{2} / \mathrm{DOF}=4.0\right)$ at $M=2.1$. The constant $c$ is consistent be-

TABLE III. Exponential fit of the anomalous quark mass $m_{5 q}$ for the RG-improved action. Strong coupling results at $\beta=2.2$ are for the lattice size $16^{3} \times 24$, and those at a weak coupling of $\beta=2.6$ are for $16^{3} \times 32$ lattice. Fits with all of the five data points are represented by the fitting range $10-50$ or 4-24. Four-points fits without the data of smallest $N_{s}$ are represented by $20-50$ or $10-24$.

\begin{tabular}{cccccccccc}
\hline \hline & \multicolumn{3}{c}{ Fitting range } & \multicolumn{4}{c}{$c+\alpha e^{-\xi N_{s}}$} \\
$\beta$ & $M$ & of $N_{s}$ & $c$ & $\alpha$ & $\xi$ & $\chi^{2} / \mathrm{DOF}$ & $\alpha$ & $\xi$ & $\chi^{2} / \mathrm{DOF}$ \\
\hline & & $10-50$ & $0.00371(46)$ & $0.0707(88)$ & $0.121(12)$ & 3.0 & $0.0454(85)$ & $0.066(11)$ & 35.7 \\
& 1.7 & $20-50$ & $0.0023(11)$ & $0.0255(72)$ & $0.062(20)$ & 0.00002 & $0.0199(16)$ & $0.0370(28)$ & 0.9 \\
2.2 & & $10-50$ & $0.00296(24)$ & $0.0355(27)$ & $0.0979(74)$ & 0.2 & $0.0254(39)$ & $0.0505(76)$ & 21.0 \\
& 2.1 & $20-50$ & $0.00278(43)$ & $0.028(11)$ & $0.085(21)$ & 0.1 & $0.0145(23)$ & $0.0328(51)$ & 4.0 \\
\hline \multirow{3}{*}{2.6} & 1.8 & $4-24$ & $0.0000099(71)$ & $0.1222(88)$ & $0.501(15)$ & 12.8 & $0.1208(98)$ & $0.498(17)$ & 16.9 \\
\hline \hline
\end{tabular}




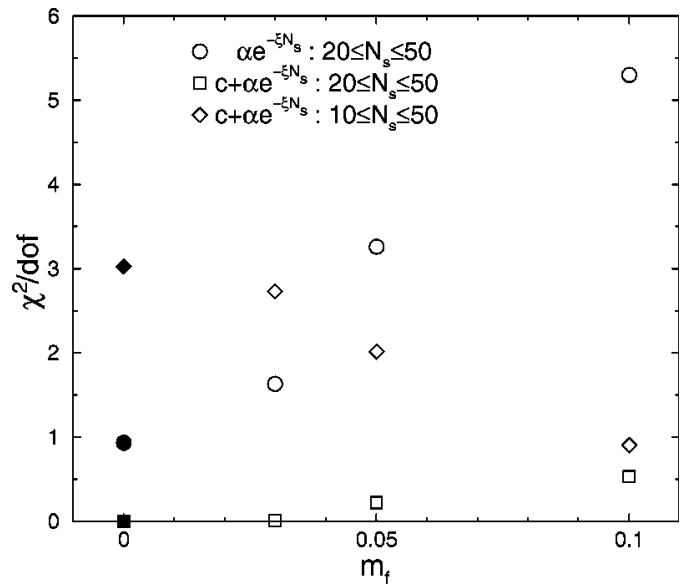

FIG. 9. $\chi^{2} / \mathrm{DOF}$ in the five and four points fit of $m_{5 q}$ data in strong coupling RG action at $\beta=2.2, M=1.7$. Open circles represent those from the four points fit with simple exponential form $\alpha e^{-\xi N_{s}}$.

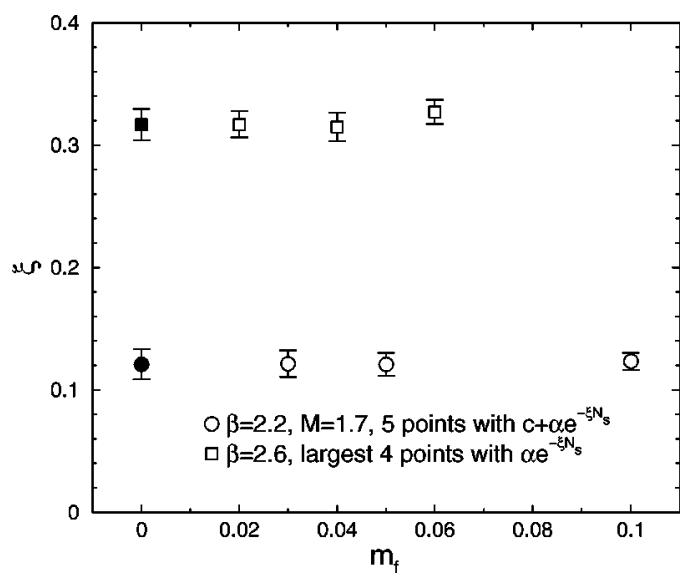

FIG. 10. Decay rate $\xi$ of the anomalous quark mass at each $m_{f}$ for the RG-improved action. Open circles represent data in the strong coupling region from the five points fit with $c+\alpha e^{-\xi N_{s}}$ at $M=1.7$. The open squares are those in the weak coupling region from the four points fit with $\alpha e^{-\xi N_{s}}$ at $M=1.8$.

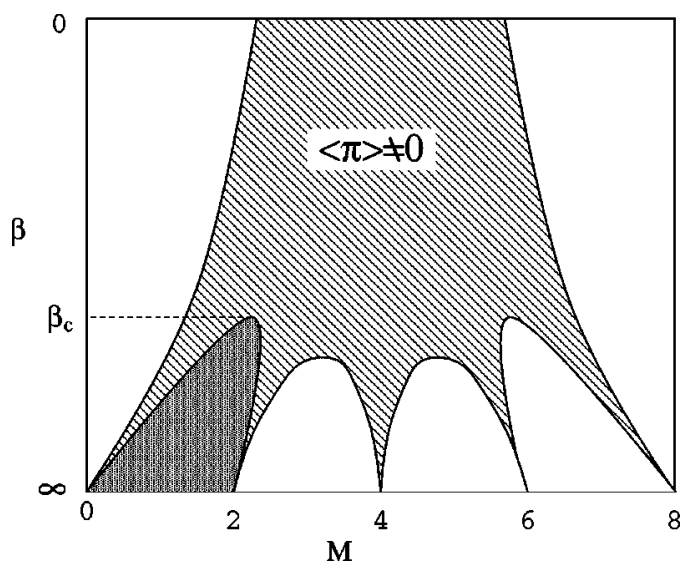

FIG. 11. Phase structure of the four dimensional Wilson fermion system. $M$ represents the fermion mass (domain-wall height). The five cusps at $\beta=\infty$ correspond to the points where subsets of 16 fermions become massless. The shaded area marked by $\langle\pi\rangle \neq 0$ represents the parity broken phase. tween the four- and five-point fits. This analysis supports theconclusion that chiral symmetry is not realized at $M$ $=2.1$. The four data points at $M=1.7$, on the other hand, are fitted well with either of the two forms with $\chi^{2} / \mathrm{DOF}<1$. The constant $c$ for the four-point fit is consistent with zero within two standard deviations. Thus the possibility that $m_{5 q}$ vanishes exponentially in $N_{s}$ at $M=1.7$ cannot be excluded from this analysis at $m_{f}=0$ alone.

To further explore this issue, we attempt to fit the data for $m_{f} \neq 0$. We find that the plain exponential does not fit the data at non-zero $m_{f}$, leading to a large $\chi^{2} / \mathrm{DOF}$ as shown in Fig. 9. Since we expect the anomalous quark mass to depend little on $m_{f}$ from the transfer matrix formalism of DWQCD, this suggests that the good fit we obtained without a constant at $m_{f}=0$ is perhaps accidental. While further data are needed for a definitive conclusion, we think it likely that a non-zero anomalous quark mass also remains in the $N_{s} \rightarrow \infty$ limit at $M=1.7$.

The value of the residual quark mass is small. From the five-point fit, we obtain

$$
m_{5 q}= \begin{cases}3.60(45) \mathrm{MeV} & \text { at } M=1.7 \\ 2.87(23) \mathrm{MeV} & \text { at } M=2.1\end{cases}
$$

We finally show the decay rates $\xi$ at finite $m_{f}$ and $m_{f}$ $=0$ in Fig. 10. The decay rates at $\beta=2.2$ are obtained by the five-point fit with a constant, while those at $\beta=2.6$ employ plain exponential fits to the four data points. As expected, the decay rates depend little on $m_{f}$; their values at $m_{f} \neq 0$ are consistent with those at $m_{f}=0$.

We conclude that the RG-improved action significantly improves the chiral behavior of DWQCD. While chiral symmetry is still not realized at $a^{-1} \simeq 1 \mathrm{GeV}$, the residual anomalous quark mass is sizably reduced compared with that with the plaquette action. The results at a weaker coupling $\left(a^{-1} \simeq 2 \mathrm{GeV}\right)$ are consistent with the vanishing anomalous quark mass, supporting the realization of chiral symmetry with DWQCD.

\section{DISCUSSION}

Let us now try to understand our findings. The anomalous quark mass $m_{5 q}$ vanishes exponentially in $N_{s}$ if the eigenvalues of the transfer matrix in the fifth direction are strictly less than unity [5]. This is also suggested from the fact that the Dirac operator of the effective theory $[10,11]$ becomes local and well defined in this case $[35,36]$. The occurrence of a unit eigenvalue, in turn, is in one-to-one correspondence with that of a zero eigenvalue of the four-dimensional Hermitian Wilson-Dirac operator $H_{W}$ [3] or more complicated one, $\hat{H}_{W}$, defined through the fifth dimensional transfer matrix $\hat{T}[5,10]$,

It has been argued that zero eigenvalues of $H_{W}$ are related to the existence of the parity-flavor broken phases in ordinary lattice QCD with the Wilson quark action [37-41]. The connection follows from the identity for the parity-flavor order parameter $\left\langle\bar{q} i \gamma_{5} \tau^{3} q\right\rangle$ given by 


$$
\begin{aligned}
\lim _{H \rightarrow+0}\left\langle\bar{q} i \gamma_{5} \tau^{3} q\right\rangle= & -\lim _{H \rightarrow+0} \operatorname{Tr} \frac{i \gamma_{5} \tau^{3}}{D_{W}+i \gamma_{5} \tau^{3} H} \\
= & -\lim _{H \rightarrow+0} \operatorname{tr}\left[\frac{i \gamma_{5}}{D_{W}+i \gamma_{5} H}-\frac{i \gamma_{5}}{D_{W}-i \gamma_{5} H}\right] \\
= & -i \lim _{H \rightarrow+0} \operatorname{tr}\left[\frac{1}{H_{W}+i H}-\frac{1}{H_{W}-i H}\right] \\
= & -i \lim _{H \rightarrow+0} \int d \lambda \rho_{H_{W}}(\lambda) \\
& \times\left\langle\lambda\left|\left(\frac{1}{\lambda+i H}-\frac{1}{\lambda-i H}\right)\right| \lambda\right\rangle \\
= & -i \int d \lambda \rho_{H_{W}}(\lambda)(-2 \pi i) \delta(\lambda) \\
= & -2 \pi \rho_{H_{W}}(0)
\end{aligned}
$$

where $H$ is an external field coupled to the order parameter, $H_{W}=\gamma_{5} D_{W}$ with $D_{W}$ the Wilson-Dirac operator, and $\rho_{H_{W}}(\lambda)$ is the density of the eigenvalues of $H_{W}$.

In Fig. 11, the expected phase diagram is schematically drawn in the $(\beta, M)$ plane, where $-M$ is the bare quark mass of the Wilson quark action. In the shaded regions the parity-flavor symmetry is broken spontaneously corresponding to a non-zero density of zero eigenvalues of $H_{W}$. At the critical lines that form the phase boundaries, the neutral pion mass vanishes. At a strong coupling $\left(\beta<\beta_{c}\right)$ there are only two critical lines, while there exist ten of them at a weak coupling $\left(\beta>\beta_{c}\right)$ and 5 points where two lines meet at $\beta$ $=\infty$. Each point corresponds to one continuum limit, whose low energy spectra are composed of a part of sixteen fermion doublers.

For DWQCD to work we have to tune the domain-wall height $M$ within the thick shaded region in Fig. 11 in order to avoid zero eigenvalues of $H_{W}$. In the weak coupling limit, this region is given by $0<M<2$. As the coupling increases, the range is shifted to a larger value of $M$ and shrinks in width. Finally no massless fermion exists in the strong coupling region at $\beta<\beta_{c}$.

If we assume that absence of a zero eigenvalue is not only a sufficient but also a necessary condition for the success of DWQCD, a naive interpretation of our results for DWQCD according to this picture would be that $\beta_{c}>6.0$ for the plaquette action while $2.6>\beta_{c}>2.2$ for the RG-improved action, or in terms of the lattice spacing $a_{c}^{-1}$ evaluated at $\beta=\beta_{c}, a_{c}^{-1} \gtrsim 2 \mathrm{GeV}$ for the former action and $2 \mathrm{GeV}$ $\gtrsim a_{c}^{-1} \gtrsim 1 \mathrm{GeV}$ for the latter.

This interpretation seems inconsistent with the quenched spectrum result for the plaquette action obtained in Ref. [40]; in this work, non-zero pion mass, signalling the parity conserving phase, was reported over the range $0.909(11) \leqslant M$ $\leqslant 2.347(5)$ at $\beta=6.0$, indicating $\beta_{c}<6.0$. A possible explanation reconciling this apparent conflict is as follows. If the order parameter, Eq. (6.1), remains non-zero in the $H$ $\rightarrow+0$ limit, the charged pions become massless NambuGoldstone modes associated with spontaneous parity-flavor breaking in the infinite volume limit. The fact that $m_{5 q}$ in the proper chiral limit is tiny at $\beta=6.0$ suggests that the magnitude of the order parameter is extremely small. Since the simulation in Ref. [40] is performed on a finite volume $\left(16^{3}\right)$ without adding the external field $H$, it may be difficult to detect the existence of massless pions corresponding to such a small order parameter. The anomalous quark mass, $m_{5 q}$, on the other hand, is very sensitive to the small eigenvalues of $H_{W}$, and hence to the non-zero order parameter. To clarify this issue, a more detailed study for the parity-flavor breaking phase of ordinary lattice QCD with the Wilson quark will be needed.

The actual dynamics inducing non-zero $\rho_{H_{W}}(0)$ may be complicated, possibly involving instantons. The relation between the spectral gap of the Hermitian Wilson-Dirac operator $H_{W}$ and the instanton number has been extensively studied [42]. While a single instanton causes a zero eigenvalue of $H_{W}$ only for a single value of $M$, an ensemble of instantons may lead to a non-zero density $\rho_{H_{W}}(0) \neq 0$. In particular unphysical short-distance topological dislocations, having an action less than that of physical instantons that are present for the plaquette action, may lead to such an effect. They will lead to an anomalous quark mass even at $a^{-1} \simeq 2 \mathrm{GeV}$ as we have found in our simulations.

This mechanism may also explain the difference in the lattice spacing needed to realize chiral symmetry in DWQCD between the plaquette and RG-improved actions. The RG-improved gauge action suppresses unphysical dislocations by pushing the action above that of physical instantons [43]. Hence their influence on the density of zero eigenvalues will be negligibly small compared to that for the plaquette action. Work exploring zero eigenvalues of the Wilson-Dirac operator for both gauge actions [44] should shed light on this interesting problem.

\section{CONCLUSIONS}

We have investigated the chiral property of domain-wall QCD within the quenched approximation. We have defined the anomalous quark mass (axial WT identity quark mass) as an indicator for the realization of chiral symmetry. Our simulations have been made in both strong and weak coupling regions corresponding to $a^{-1} \simeq 1$ and $2 \mathrm{GeV}$, using the plaquette and RG-improved gauge actions. We have found that the anomalous quark mass remains non-zero in the DWQCD chiral limit, $m_{f} \rightarrow 0$ and $N_{s} \rightarrow \infty$ for the plaquette action for $a^{-1} \leqslant 2 \mathrm{GeV}$ within our numerical data taken and the assumption of a single exponential plus a constant for the form of fit function. The magnitude of chiral symmetry breaking rapidly decreases with lattice spacing, but the exact chiral symmetry is not realized at least for this lattice spacing.

On the contrary, our analysis for the RG-improved action reveals a much improved chiral behavior. The anomalous quark mass vanishes exponentially with this action at $a^{-1}$ $\simeq 2 \mathrm{GeV}$, indicating that the exact chiral symmetry is real- 
ized. At $a^{-1} \simeq 1 \mathrm{GeV}$ the improvement of the chiral behavior is not sufficient to remove a non-zero anomalous quark mass.

Overall, quenched domain-wall QCD at $a^{-1} \simeq 1 \mathrm{GeV}$ appears no better than usual lattice QCD with the ordinary Wilson quark action. Since the effect of explicit chiral symmetry breaking is non-negligible, the operators relevant for the electroweak matrix elements mix nontrivially between different chiralities. There is no a priori reason to expect that these mixing coefficients are small.

The situation is better in the weak coupling region, e.g., at $a^{-1} \simeq 2 \mathrm{GeV}$. Even with the plaquette action, the chiral symmetry breaking effect is significantly smaller than the physical $u, d$ quark masses, and hence may not seriously affect the chiral properties of weak matrix elements [20]. Moreover, the chiral property of DWQCD is distinctly improved with the RG action: not only the explicit breaking is shown to vanish exponentially in $N_{s}$, but also the size of the breaking effect at finite $N_{s}$ is smaller. The improved gauge actions, such as the RG-improved one, should be a preferred choice for future numerical simulations for DWQCD.

\section{ACKNOWLEDGMENTS}

This work is supported in part by the Grants-in-Aid of Ministry of Education (Nos. 09304029, 10640246, $10640248,11640250,10740107,11640250,11640294$, $11740162,12640253,12014202,2373)$. A.A.K. and T.M. are supported by the JSPS Research for the Future Program (No. JSPS-RFTF 97P01102). S.E., K.N., and J.N. are supported by JSPS.

\section{APPENDIX A: PION MASS}

The pion mass is a standard quantity in examining the chiral property, and hence often studied in the context of DWQCD. Analyses of this observable, however, is not straightforward due to possible finite size effects, particularly for small pion masses, and difficulties associated with the chiral extrapolation because of chiral logarithms, which may be quite significant in quenched QCD. Numerically the pion mass has to be extracted from an exponential falloff of the pion propagator. Even though the pion propagator has the best statistical quality among hadron propagators, this procedure is more susceptible to statistical and systematic uncertainties than the determination of the anomalous quark mass which involves only a constant fit to a ratio of two kinds of propagators. For these reasons we have employed the anomalous quark mass in the main body of the present paper. In this appendix we present our results for the pion mass, and discuss to what extent they match with those obtained with the anomalous quark mass.

Typical results for pion mass squared $m_{\pi}^{2}\left(m_{f}, N_{s}, M\right)$ are plotted as a function of the averaged bare quark mass $m_{f}^{a v}$ $=\left(m_{f}^{1}+m_{f}^{2}\right) / 2$ for the plaquette action in Fig. 12. The result shows a good linear behavior in $m_{f}^{a v}$. The pion mass in the chiral limit $m_{f} \rightarrow 0$ is therefore estimated by linearly extrapolating $m_{\pi}^{2}$ in $m_{f}^{a v}$. The numerical values of the pion mass are collected in Appendix B.

\section{Strong coupling region}

We first discuss pion mass results at the strong coupling $a^{-1} \simeq 1 \mathrm{GeV}$. In Fig. 13(a) we plot the pion mass squared $m_{\pi}^{2}$ in the chiral limit $m_{f}^{a v}=0$ as a function of $N_{s}$ for the plaquette action at $\beta=5.65$. Filled symbols are obtained on a lattice of spatial size $N_{\sigma}=16$ and open ones with a spatial size of $N_{\sigma}=12$. A similar figure for the RG-improved action is shown in Fig. 13(b).

Solid lines in these figures show results of fits by a form $c+\alpha e^{-\xi N_{s}}$ using all five points obtained on an $N_{\sigma}=16$ lattice $(M=1.7,2.1)$ or an $N_{\sigma}=12$ lattice $(M=1.3,2.5)$. They reproduce the data well for a non-zero $c$ with an acceptable $\chi^{2}$ as summarized in Tables IV and V. Therefore pion mass does not vanish in the chiral limit $m_{f} \rightarrow 0$ and $N_{s} \rightarrow \infty$ at $a^{-1} \simeq 1 \mathrm{GeV}$ for the plaquette and the RG-improved actions, which is consistent with the conclusions from the analysis of the anomalous quark mass.

In order to check our procedure of taking the chiral limit we interchange the order of the limits $m_{f}^{a v} \rightarrow 0$ and $N_{s} \rightarrow \infty$. As shown by solid lines going through open circles in Fig. 14 , we first make a fit of form $m_{\pi}^{2}\left(m_{f}^{a v}, N_{s}\right)=c^{\prime}\left(m_{f}^{a v}\right)$ $+\alpha e^{-\xi N_{s}}$ for each value of $m_{f}^{a v}$. We note that the constant term has to be included in the fit of the pion mass at non-zero $m_{f}$. A linear chiral extrapolation $m_{\pi}^{2}\left(m_{f}^{a v}, N_{s}=\infty\right)$ $=c^{\prime}\left(m_{f}^{a v}\right)=d+\gamma m_{f}^{a v}$ then yields $d=0.0432(52)$, which agrees well with the value of $c=0.0440$ (43) previously obtained. The commutativity of the two limits is summarized by two symbols " $\mathrm{X}$ " and " $\mathrm{Y}$ " in Fig. 14, where $\mathrm{X}$ represents $m_{\pi}^{2}\left(m_{f}^{a v}=0, N_{s}=\infty\right)$ given with $m_{f} \rightarrow 0$, then $N_{s} \rightarrow \infty$ and $\mathrm{Y}$ represents the pion mass with the limit $N_{s} \rightarrow \infty$, then $m_{f} \rightarrow 0$. These two values agree very well with each other. The same consistency check is also made for the RGimproved action.

The above analyses strongly support the conclusion that $m_{\pi}$ does not vanish in the limit $m_{f}^{a v} \rightarrow 0$ and $N_{s} \rightarrow \infty$. In order to examine finite spatial volume effects as a possible origin of this non-zero mass, we compare the pion mass squared in the proper chiral limit $m_{\pi}^{2}\left(m_{f}^{a v} \rightarrow 0, N_{s} \rightarrow \infty\right)$ for two kinds of lattice volumes $12^{3} \times 24$ (open symbols) and $16^{3} \times 24$ (filled symbols) in Fig. 15.

The circles and diamonds represent the results from the plaquette and the RG action, respectively. The horizontal triangles are results for the Nambu-Goldstone pion mass of the Kogut-Susskind quark action at $\beta=5.7\left(a^{-1}=1.12\right.$ $\mathrm{GeV}$ ) for a spatial lattice size of $N_{\sigma}=12$ (open triangle) [45] and $N_{\sigma}=16$ (filled triangle) [46]. From comparison of these data points, we consider that there may be some finite size effects at $M=1.7$, but that they are not large enough to explain non-zero values of pion mass for DWQCD at strong coupling.

Since there still remains a possibility that this non-zero mass is caused by the quenching effect, we adopt the WT identity mass as a better indicator to test the chiral property of DWQCD in the main body of this article.

\section{Weak coupling region}

Pion mass squared in the chiral limit $m_{\pi}^{2}\left(m_{f}=0, N_{s}, M\right)$ is plotted in Fig. 16 for the plaquette action at $\beta=6.0$ and in 

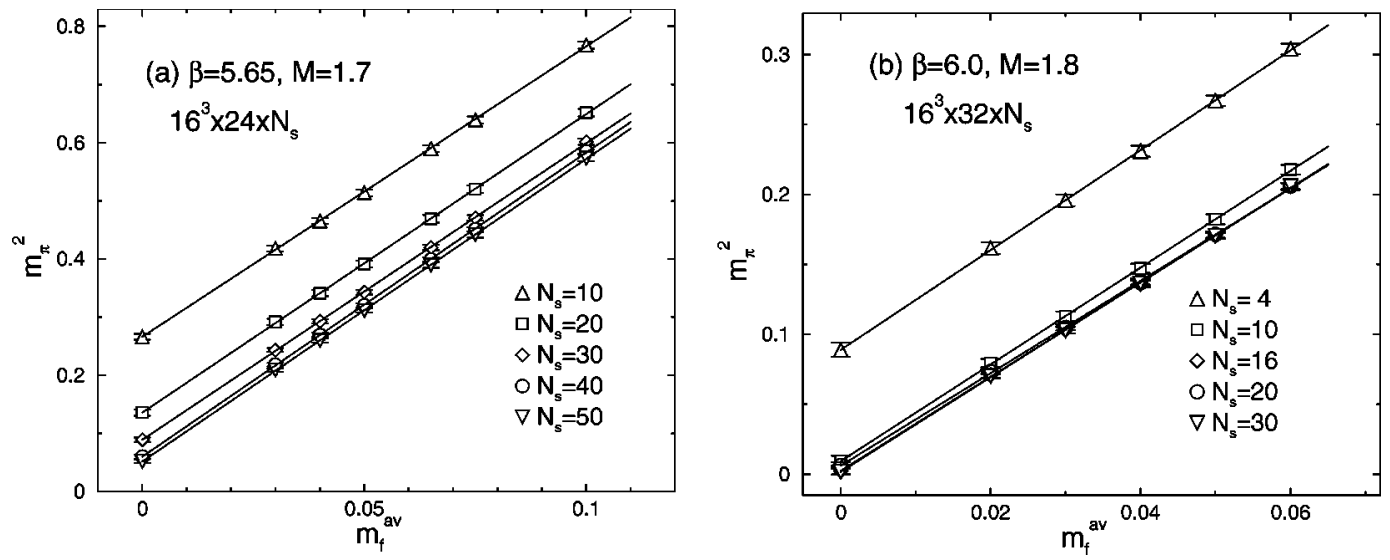

FIG. 12. Pion mass squared as a function of valence quark mass $m_{f}$ at $M=1.7, \beta=5.65$ on $16^{3} \times 24 \times N_{s}$ lattice (a) and $\beta=6.0$ on $16^{3} \times 32 \times N_{s}$ lattice (b) of plaquette action. Lines are linear fits to those data.
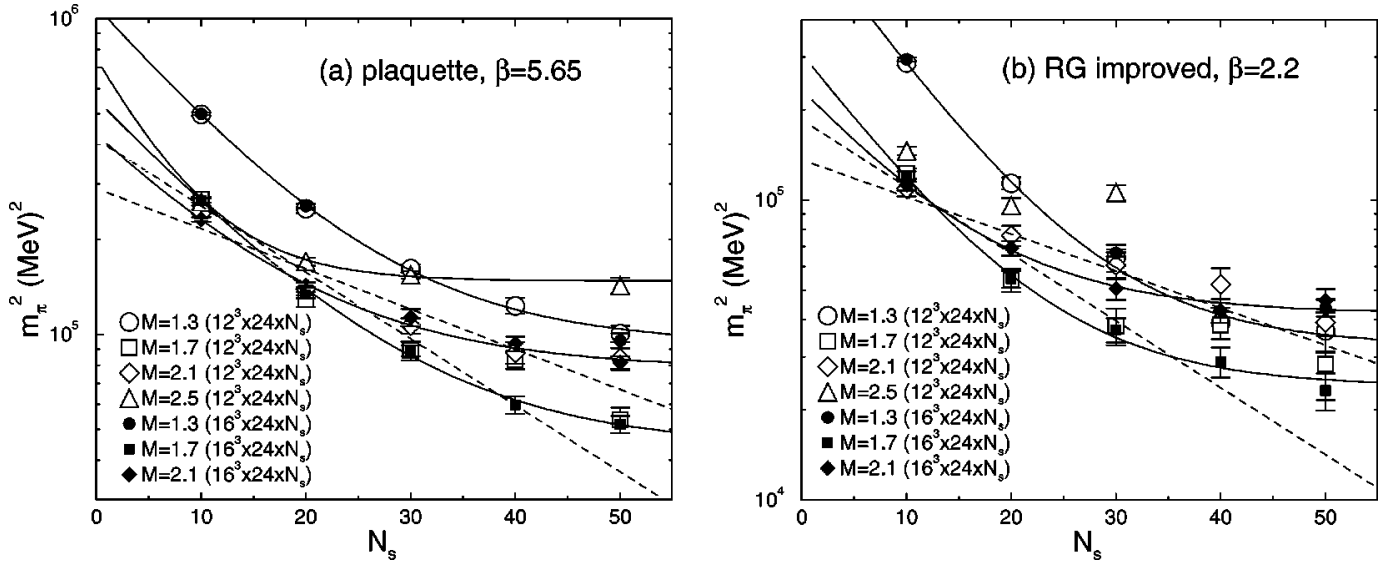

FIG. 13. Pion mass squared at $m_{f}=0$ as a function of the extra dimension $N_{s}$ in the strong coupling region, $\beta=5.65$ for plaquette action (a) and $\beta=2.2$ for RG-improved action (b). Filled symbols are taken on $16^{3} \times 24 \times N_{s}$ lattice, and open ones on $12^{3} \times 24 \times N_{s}$ lattice. Lines are fits to an exponential with or without a constant of all five data points on an $N_{\sigma}=16$ lattice except for $M=1.3$ (a),(b) and 2.5 (a) for which data fitted are for $N_{\sigma}=12$.
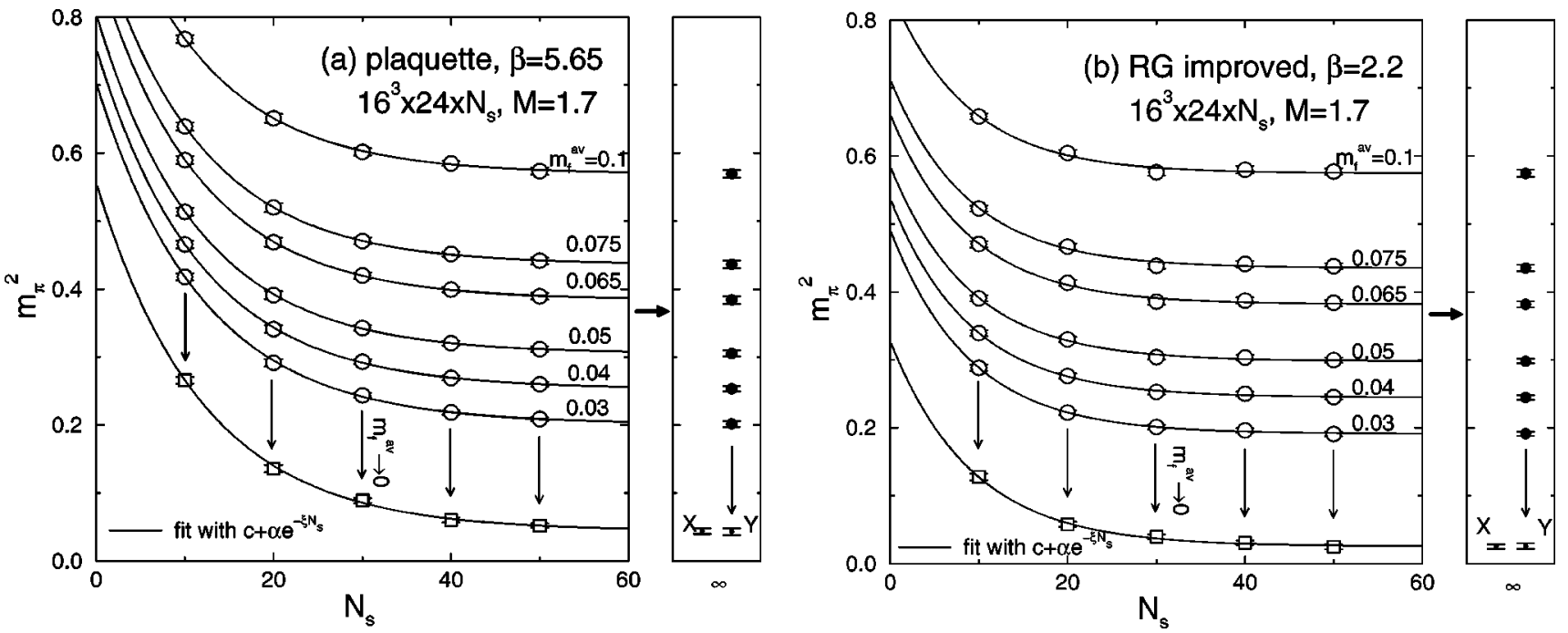

FIG. 14. Pion mass squared as a function of the extra dimension $N_{s}$ at $M=1.7$ on $16^{3} \times 24 \times N_{s}$ lattice. Figure (a) represents the results for the plaquette action at $\beta=5.65$. Figure (b) is for the RG action at $\beta=2.2$. The pion masses derived with two different order of limits $m_{f} \rightarrow 0, N_{s} \rightarrow \infty$ are represented with " $\mathrm{X}$ "' and "Y."' 
TABLE IV. Exponential fit of pion mass squared $m_{\pi}^{2}$ in strong coupling region at $M=1.7$ and 2.1. The four-dimensional lattice size is $16^{3} \times 24$. Fits with all of five data points are represented by the fitting range $10-50$ and four-points fits without $N_{s}=10$ data are represented by $20-50$.

\begin{tabular}{|c|c|c|c|c|c|c|c|c|c|}
\hline \multirow[b]{2}{*}{$\beta$} & \multirow[b]{2}{*}{$M$} & \multirow{2}{*}{$\begin{array}{c}\text { Fitting range } \\
\text { of } N_{s}\end{array}$} & \multicolumn{4}{|c|}{$c+\alpha e^{-\xi N_{s}}$} & \multicolumn{3}{|c|}{$\alpha e^{-\xi N_{s}}$} \\
\hline & & & $c$ & $\alpha$ & $\xi$ & $\chi^{2} / \mathrm{DOF}$ & $\alpha$ & $\xi$ & $\chi^{2} / \mathrm{DOF}$ \\
\hline \multirow{4}{*}{5.65} & & $10-50$ & $0.0440(43)$ & $0.511(30)$ & $0.0836(62)$ & 0.8 & $0.415(47)$ & $0.0485(54)$ & 16.1 \\
\hline & 1.7 & $20-50$ & $0.0395(83)$ & $0.40(11)$ & $0.071(16)$ & 0.9 & $0.257(39)$ & $0.0341(46)$ & 3.6 \\
\hline & & $10-50$ & $0.0780(46)$ & $0.352(25)$ & $0.0832(78)$ & 0.8 & $0.290(33)$ & $0.0293(54)$ & 20.4 \\
\hline & 2.1 & $20-50$ & $0.060(26)$ & $0.207(54)$ & $0.046(28)$ & 0.004 & $0.211(10)$ & $0.0195(16)$ & 0.5 \\
\hline \multirow{4}{*}{2.2} & & $10-50$ & $0.0255(32)$ & $0.301(44)$ & $0.109(14)$ & 0.6 & $0.196(36)$ & $0.0513(92)$ & 8.6 \\
\hline & 1.7 & $20-50$ & $0.019(10)$ & $0.137(77)$ & $0.063(38)$ & 0.07 & $0.103(14)$ & $0.0300(46)$ & 0.5 \\
\hline & & $10-50$ & $0.0445(43)$ & $0.204(41)$ & $0.100(21)$ & 0.6 & $0.145(23)$ & $0.0285(66)$ & 7.2 \\
\hline & 2.1 & $20-50$ & $0.0467(45)$ & $0.5(1.1)$ & $0.15(11)$ & 0.7 & $0.098(19)$ & $0.0169(59)$ & 2.8 \\
\hline
\end{tabular}

TABLE V. Exponential fit of the pion mass squared $m_{\pi}^{2}$ in strong coupling region at $M=1.3,1.7,2.1,2.5$. The four dimensional lattice size is $12^{3} \times 24$. Fits with all of five data points are represented by the fitting range $10-50$ and four-points fit without $N_{s}=10$ data are represented by $20-50$.

\begin{tabular}{|c|c|c|c|c|c|c|c|c|c|}
\hline \multirow[b]{2}{*}{$\beta$} & \multirow[b]{2}{*}{$M$} & \multirow{2}{*}{$\begin{array}{c}\text { Fitting range } \\
\text { of } N_{s}\end{array}$} & \multicolumn{4}{|c|}{$c+\alpha e^{-\xi N_{s}}$} & \multicolumn{3}{|c|}{$\alpha e^{-\xi N_{s}}$} \\
\hline & & & $c$ & $\alpha$ & $\xi$ & $\chi^{2} / \mathrm{DOF}$ & $\alpha$ & $\xi$ & $\chi^{2} / \mathrm{DOF}$ \\
\hline & & $10-50$ & $0.0933(71)$ & $0.997(53)$ & $0.0904(57)$ & 0.7 & $0.78(10)$ & $0.0500(64)$ & 27.0 \\
\hline & 1.3 & $20-50$ & $0.084(13)$ & $0.75(18)$ & $0.075(14)$ & 0.1 & $0.447(70)$ & $0.0320(50)$ & 5.2 \\
\hline & & $10-50$ & $0.059(12)$ & $0.56(13)$ & $0.100(25)$ & 5.3 & $0.403(55)$ & $0.0454(74)$ & 16.6 \\
\hline & 1.7 & $20-50$ & $-0.02(28)$ & $0.23(17)$ & $0.021(56)$ & 4.9 & $0.216(35)$ & $0.0267(49)$ & 2.5 \\
\hline \multirow[t]{6}{*}{5.65} & & $10-50$ & $0.0811(66)$ & $0.429(57)$ & $0.098(14)$ & 0.2 & $0.313(45)$ & $0.0332(65)$ & 11.7 \\
\hline & 2.1 & $20-50$ & $0.077(14)$ & $0.30(20)$ & $0.077(42)$ & 0.2 & $0.198(22)$ & $0.0189(35)$ & 1.5 \\
\hline & 2.5 & $10-50$ & $0.1478(57)$ & $0.56(17)$ & $0.159(33)$ & 0.6 & $0.291(57)$ & $0.0205(86)$ & 30.2 \\
\hline & & $10-50$ & $0.0348(49)$ & $0.828(66)$ & $0.1125(84)$ & 0.4 & $0.635(86)$ & $0.0757(98)$ & 11.4 \\
\hline & 1.3 & $20-50$ & $0.0318(97)$ & $0.63(37)$ & $0.098(34)$ & 0.7 & $0.307(56)$ & $0.0475(69)$ & 2.2 \\
\hline & & $10-50$ & $0.0368(39)$ & $0.42(13)$ & $0.150(31)$ & 0.7 & $0.196(39)$ & $0.048(11)$ & 9.1 \\
\hline \multirow[t]{3}{*}{2.2} & 1.7 & $20-50$ & $0.033(15)$ & $0.12(23)$ & $0.08(12)$ & 1.3 & $0.082(15)$ & $0.0189(60)$ & 0.8 \\
\hline & & $10-50$ & $0.034(18)$ & $0.134(19)$ & $0.051(25)$ & 0.3 & $0.146(11)$ & $0.0262(35)$ & 0.5 \\
\hline & 2.1 & $20-50$ & $-0.03(23)$ & $0.15(21)$ & $0.013(35)$ & 0.2 & $0.123(21)$ & $0.0209(56)$ & 0.09 \\
\hline
\end{tabular}

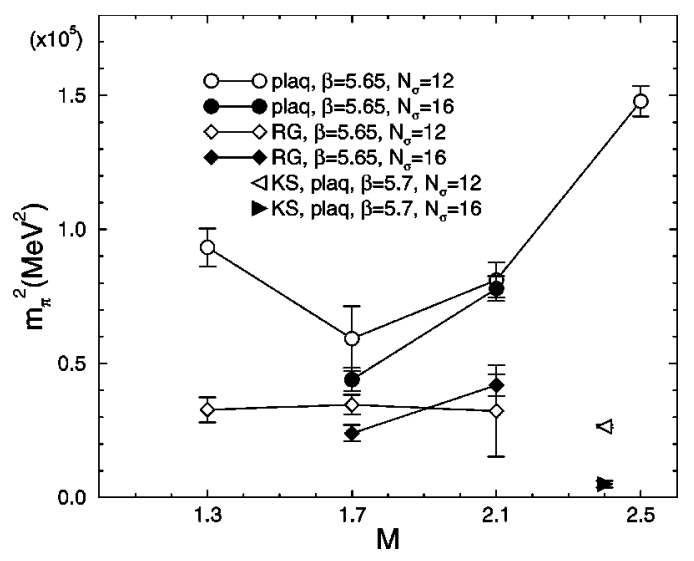

FIG. 15. Pion mass squared as a function of domain-wall height $M$ in the chiral limit $m_{f} \rightarrow 0, N_{s} \rightarrow \infty$. The results on $16^{3} \times 24$ lattice (filled symbols) are compared with those on $12^{3} \times 24$ (open symbols). Open and filled triangles are those from the KS fermion action at $N_{\sigma}=12$ [45] and $N_{\sigma}=16$ [46] lattice volumes, which represent the pure finite volume effect.

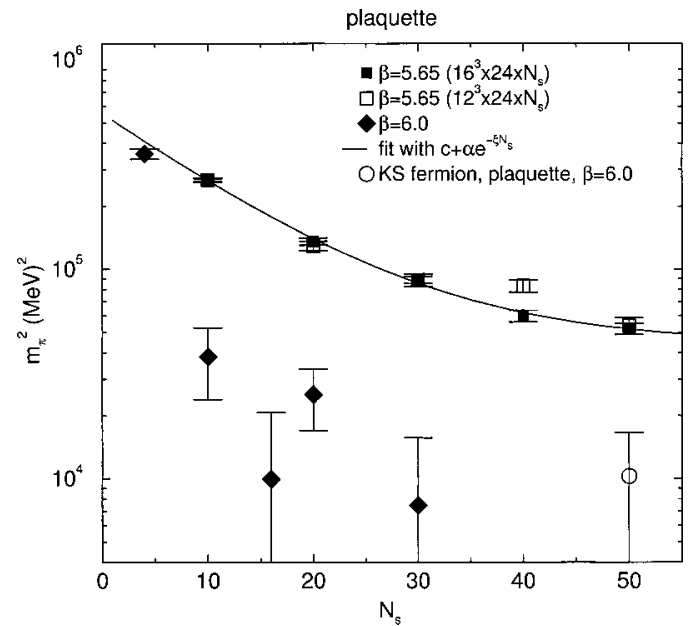

FIG. 16. Pion mass squared at $m_{f}=0$ as a function of $N_{s}$ at $\beta$ $=6.0$ of plaquette action with $M=1.8$ (filled diamonds) as compared with those at $\beta=5.65$ and and $M=1.7$ (squares). Open circle represents the pion mass from the KS fermion at $N_{\sigma}=16$ lattice volume. 


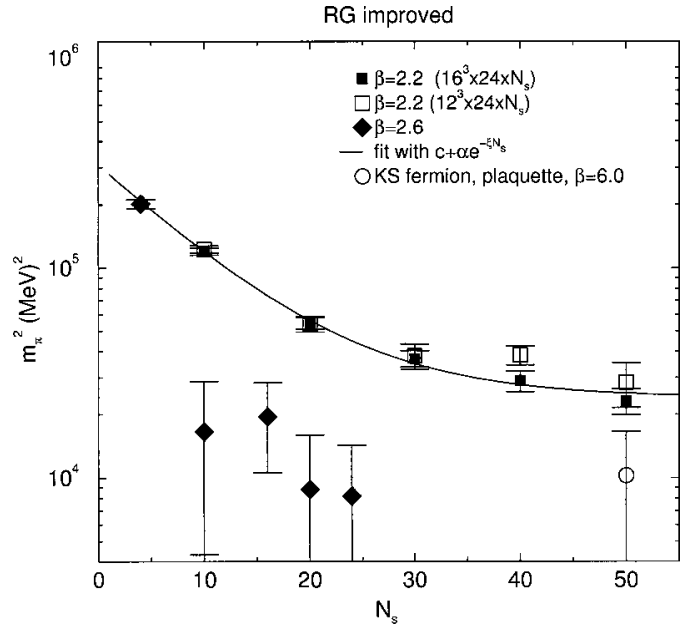

FIG. 17. Pion mass squared at $m_{f}=0$ as a function of $N_{s}$ at $\beta$ $=2.6$ of RG-improved action with $M=1.8$ (filled diamonds) as compared with those at $\beta=2.2$ and and $M=1.7$ (squares). Open circle represents the pion mass from the KS fermion at $N_{\sigma}=16$ lattice volume.

Fig. 17 for the RG-improved action at $\beta=2.6$. The results have large errors, and do not show a clear trend as a function of $N_{s}$. However, the magnitude beyond $N_{s}=10$ are comparable to the value obtained for the Nambu-Goldstone pion mass of the Kogut-Susskind fermion at $\beta=6.0$ of plaquette gauge action for the same spatial lattice size $16^{3} \times 40$ (star) [47]. This indicates that these non-zero pion masses are mainly caused by finite spatial volume effects. Hence the chiral property of DWQCD cannot be studied by pion mass in the weak coupling region on this volume.
TABLE VI. Anomalous quark mass for the plaquette action in the strong coupling region at $\beta=5.65, M=1.3,1.7,2.1$ on $12^{3} \times 24$ lattice.

\begin{tabular}{|c|c|c|c|c|c|}
\hline \multirow[t]{2}{*}{$m_{f}$} & \multicolumn{5}{|c|}{$N_{s}$} \\
\hline & 10 & 20 & 30 & 40 & 50 \\
\hline & \multicolumn{5}{|c|}{$\beta=5.65, M=1.3$ on $12^{3} \times 24$} \\
\hline 0.00 & - & - & - & $0.0605(31)$ & $0.0499(20)$ \\
\hline 0.03 & - & - & - & $0.0622(28)$ & $0.0520(19)$ \\
\hline 0.05 & - & - & - & $0.0629(26)$ & $0.0530(19)$ \\
\hline \multirow[t]{2}{*}{0.1} & - & - & - & $0.0658(24)$ & $0.0565(21)$ \\
\hline & \multicolumn{5}{|c|}{$\beta=5.65, M=1.7$ on $12^{3} \times 24$} \\
\hline 0.00 & - & - & - & $0.01303(56)$ & $0.01019(47)$ \\
\hline 0.03 & - & - & - & $0.01304(53)$ & $0.01019(42)$ \\
\hline 0.05 & - & - & - & $0.01302(59)$ & $0.01016(42)$ \\
\hline \multirow[t]{2}{*}{0.1} & - & - & - & $0.01302(63)$ & $0.01018(44)$ \\
\hline & \multicolumn{5}{|c|}{$\beta=5.65, M=2.1$ on $12^{3} \times 24$} \\
\hline 0.00 & - & - & - & $0.00934(28)$ & $0.00770(42)$ \\
\hline 0.03 & - & - & - & $0.01031(31)$ & $0.00894(29)$ \\
\hline 0.05 & - & - & - & $0.01051(35)$ & $0.00907(33)$ \\
\hline 0.1 & - & - & - & $0.01230(55)$ & $0.01155(64)$ \\
\hline
\end{tabular}

\section{APPENDIX B}

In this appendix we list results for anomalous quark mass $m_{5 q}$ and pion mass squared $m_{\pi}^{2}$. All errors are estimated with the jackknife method. See Tables VI-XVII.

TABLE VII. Anomalous quark mass for the plaquette action in the strong coupling region at $\beta=5.65, M=1.3,1.7,2.1$ on $16^{3} \times 24$ lattice.

\begin{tabular}{|c|c|c|c|c|c|}
\hline \multirow[t]{3}{*}{$m_{f}$} & \multicolumn{5}{|c|}{$N_{s}$} \\
\hline & 10 & 20 & 30 & 40 & 50 \\
\hline & \multicolumn{5}{|c|}{$\beta=5.65, M=1.3$ on $16^{3} \times 24$} \\
\hline 0.00 & $0.1747(16)$ & $0.1129(14)$ & - & - & $0.04925(98)$ \\
\hline 0.03 & $0.1723(16)$ & $0.1123(16)$ & - & - & $0.0513(11)$ \\
\hline 0.05 & $0.1700(17)$ & $0.1111(17)$ & - & - & $0.0522(11)$ \\
\hline \multirow[t]{2}{*}{0.1} & $0.1660(18)$ & $0.1102(20)$ & - & - & $0.0557(14)$ \\
\hline & \multicolumn{5}{|c|}{$\beta=5.65, M=1.7$ on $16^{3} \times 24$} \\
\hline 0.00 & $0.05514(49)$ & $0.02717(62)$ & $0.01748(55)$ & $0.01303(27)$ & $0.01095(47)$ \\
\hline 0.03 & $0.05373(46)$ & $0.02646(58)$ & $0.01721(51)$ & $0.01280(29)$ & $0.01092(39)$ \\
\hline 0.05 & $0.05228(49)$ & $0.02561(59)$ & $0.01691(51)$ & $0.01252(35)$ & $0.01084(43)$ \\
\hline \multirow[t]{2}{*}{0.1} & $0.05002(67)$ & $0.02445(60)$ & $0.01645(54)$ & $0.01219(53)$ & $0.01080(44)$ \\
\hline & \multicolumn{5}{|c|}{$\beta=5.65, M=2.1$ on $16^{3} \times 24$} \\
\hline 0.00 & $0.03349(31)$ & $0.01820(37)$ & $0.01253(39)$ & $0.00954(24)$ & $0.00757(43)$ \\
\hline 0.03 & $0.03338(26)$ & $0.01941(35)$ & $0.01358(37)$ & $0.01032(22)$ & $0.00880(38)$ \\
\hline 0.05 & $0.03261(28)$ & $0.01947(38)$ & $0.01361(37)$ & $0.01032(24)$ & $0.00909(46)$ \\
\hline 0.1 & $0.03254(37)$ & $0.02171(56)$ & $0.01542(44)$ & $0.01203(56)$ & $0.01124(51)$ \\
\hline
\end{tabular}


TABLE VIII. Anomalous quark mass for the plaquette action in the weak coupling region at $\beta=6.0, M=1.8$ on $16^{3} \times 32$ lattice.

\begin{tabular}{|c|c|c|c|c|c|}
\hline$m_{f}$ & 4 & 10 & $\begin{array}{c}N_{s} \\
16\end{array}$ & 20 & 30 \\
\hline 0.0 & $0.02955(29)$ & $0.00373(13)$ & $0.00148(17)$ & $0.00084(11)$ & $0.000467(57)$ \\
\hline 0.02 & $0.02884(25)$ & $0.00360(11)$ & $0.00134(14)$ & $0.000750(90)$ & $0.000403(49)$ \\
\hline 0.04 & $0.02804(20)$ & $0.003446(78)$ & $0.001128(93)$ & $0.000637(73)$ & $0.000317(42)$ \\
\hline 0.06 & $0.02735(18)$ & $0.003316(64)$ & $0.000989(66)$ & $0.000548(59)$ & $0.000259(38)$ \\
\hline
\end{tabular}

TABLE IX. Anomalous quark mass for the RG improved action in the strong coupling region at $\beta=2.2, M=1.3,1.7,2.1$ on $12^{3} \times 24$ lattice.

\begin{tabular}{|c|c|c|c|c|c|}
\hline \multirow[t]{2}{*}{$m_{f}$} & \multicolumn{5}{|c|}{$N_{s}$} \\
\hline & 10 & 20 & 30 & 40 & 50 \\
\hline & \multicolumn{5}{|c|}{$\beta=2.2, M=1.3$ on $12^{3} \times 24$} \\
\hline 0.00 & - & - & - & $0.01565(90)$ & $0.01260(81)$ \\
\hline 0.03 & - & - & - & $0.01605(84)$ & $0.01324(78)$ \\
\hline 0.05 & - & - & - & $0.01657(86)$ & $0.01385(80)$ \\
\hline \multirow[t]{2}{*}{0.1} & - & - & - & $0.0172(11)$ & $0.01491(84)$ \\
\hline & \multicolumn{5}{|c|}{$\beta=2.2, M=1.7$ on $12^{3} \times 24$} \\
\hline 0.00 & - & - & - & $0.00413(23)$ & $0.00319(26)$ \\
\hline 0.03 & - & - & - & $0.00399(20)$ & $0.00302(24)$ \\
\hline 0.05 & - & - & - & $0.00402(24)$ & $0.00286(25)$ \\
\hline \multirow[t]{2}{*}{0.1} & - & - & - & $0.00377(21)$ & $0.00257(26)$ \\
\hline & \multicolumn{5}{|c|}{$\beta=2.2, M=2.1$ on $12^{3} \times 24$} \\
\hline 0.00 & - & - & - & $0.00378(25)$ & $0.00303(24)$ \\
\hline 0.03 & - & - & - & $0.00386(21)$ & $0.00306(19)$ \\
\hline 0.05 & - & - & - & $0.00373(22)$ & $0.00299(17)$ \\
\hline 0.1 & - & - & - & $0.00388(23)$ & $0.00304(24)$ \\
\hline
\end{tabular}

TABLE X. Anomalous quark mass for the RG improved action in the strong coupling region at $\beta=2.2, M=1.3,1.7,2.1$ on $16^{3} \times 24$ lattice.

\begin{tabular}{|c|c|c|c|c|c|}
\hline$m_{f}$ & \multicolumn{5}{|c|}{$N_{s}$} \\
\hline & \multicolumn{5}{|c|}{$\beta=2.2, M=1.3$ on $16^{3} \times 24$} \\
\hline 0.00 & $0.09728(99)$ & - & $0.02339(41)$ & - & $0.01329(48)$ \\
\hline 0.03 & $0.0950(10)$ & - & $0.02345(39)$ & - & $0.01356(49)$ \\
\hline 0.05 & $0.0928(11)$ & - & $0.02339(41)$ & - & $0.01378(59)$ \\
\hline \multirow[t]{2}{*}{0.1} & $0.0890(11)$ & - & $0.02350(55)$ & - & $0.01422(74)$ \\
\hline & \multicolumn{5}{|c|}{$\beta=2.2, M=1.7$ on $16^{3} \times 24$} \\
\hline 0.00 & $0.02486(42)$ & $0.00966(35)$ & $0.00626(39)$ & $0.00442(28)$ & $0.00344(33)$ \\
\hline 0.03 & $0.02387(38)$ & $0.00920(32)$ & $0.00586(35)$ & $0.00412(26)$ & $0.00329(28)$ \\
\hline 0.05 & $0.02281(35)$ & $0.00875(33)$ & $0.00549(33)$ & $0.00379(23)$ & $0.00321(22)$ \\
\hline \multirow[t]{2}{*}{0.1} & $0.02113(35)$ & $0.00800(32)$ & $0.00480(33)$ & $0.00326(24)$ & $0.00296(20)$ \\
\hline & \multicolumn{5}{|c|}{$\beta=2.2, M=2.1$ on $16^{3} \times 24$} \\
\hline 0.00 & $0.01633(35)$ & $0.00788(30)$ & $0.00490(26)$ & $0.00377(25)$ & $0.00314(22)$ \\
\hline 0.03 & $0.01626(29)$ & $0.00804(28)$ & $0.00510(21)$ & $0.00381(19)$ & $0.00318(18)$ \\
\hline 0.05 & $0.01580(30)$ & $0.00782(29)$ & $0.00501(20)$ & $0.00368(18)$ & $0.00312(16)$ \\
\hline 0.1 & $0.01570(28)$ & $0.00811(30)$ & $0.00535(21)$ & $0.00374(20)$ & $0.00317(19)$ \\
\hline
\end{tabular}


TABLE XI. Anomalous quark mass for the RG improved action in the weak coupling region at $\beta=2.6, M=1.8$ on $16^{3} \times 32$ lattice.

\begin{tabular}{lccccc}
\hline \hline$m_{f}$ & & & $N_{s}$ & & \\
& 4 & 10 & 16 & 20 & 24 \\
\hline 0.0 & $0.01649(11)$ & $0.000817(20)$ & $0.000146(22)$ & $0.000042(11)$ & $0.000009(2)$ \\
0.02 & $0.016238(99)$ & $0.000812(17)$ & $0.000136(17)$ & $0.0000405(87)$ & $0.0000087(15)$ \\
0.04 & $0.015920(80)$ & $0.000803(16)$ & $0.000116(12)$ & $0.0000397(78)$ & $0.0000090(19)$ \\
0.06 & $0.015677(70)$ & $0.000801(16)$ & $0.0001060(76)$ & $0.0000387(73)$ & $0.0000089(19)$ \\
\hline \hline
\end{tabular}

TABLE XII. Pion mass squared for the plaquette action in the strong coupling region at $\beta=5.65, M=1.3,1.7,2.1,2.5$ on $12^{3} \times 24$ lattice.

\begin{tabular}{|c|c|c|c|c|c|}
\hline \multirow[t]{2}{*}{$m_{f}$} & \multicolumn{5}{|c|}{$N_{s}$} \\
\hline & \multicolumn{5}{|c|}{$\beta=5.65, M=1.3$ on $12^{3} \times 24$} \\
\hline 0.00 & $0.4982(80)$ & $0.2504(83)$ & $0.1618(47)$ & $0.1234(71)$ & $0.1007(59)$ \\
\hline 0.03 & $0.5817(81)$ & $0.3197(82)$ & $0.2258(45)$ & $0.1845(66)$ & $0.1597(61)$ \\
\hline 0.04 & $0.6078(81)$ & $0.3415(82)$ & $0.2462(44)$ & $0.2038(63)$ & $0.1787(64)$ \\
\hline 0.05 & $0.6341(82)$ & $0.3635(83)$ & $0.2666(43)$ & $0.2233(61)$ & $0.1978(66)$ \\
\hline 0.065 & $0.6760(83)$ & $0.3981(84)$ & $0.2987(44)$ & $0.2536(61)$ & $0.2272(71)$ \\
\hline 0.075 & $0.7030(83)$ & $0.4206(84)$ & $0.3195(43)$ & $0.2736(59)$ & $0.2466(72)$ \\
\hline \multirow[t]{2}{*}{0.1} & $0.7733(84)$ & $0.4789(85)$ & $0.3733(43)$ & $0.3249(59)$ & $0.2964(76)$ \\
\hline & \multicolumn{5}{|c|}{$\beta=5.65, M=1.7$ on $12^{3} \times 24$} \\
\hline 0.00 & $0.2675(55)$ & $0.1292(68)$ & $0.0889(61)$ & $0.0833(55)$ & $0.0537(50)$ \\
\hline 0.03 & $0.4190(55)$ & $0.2840(61)$ & $0.2440(53)$ & $0.2384(54)$ & $0.2114(50)$ \\
\hline 0.04 & $0.4667(57)$ & $0.3329(61)$ & $0.2927(53)$ & $0.2882(54)$ & $0.2630(54)$ \\
\hline 0.05 & $0.5148(59)$ & $0.3822(61)$ & $0.3416(52)$ & $0.3375(52)$ & $0.3147(58)$ \\
\hline 0.065 & $0.5912(62)$ & $0.4589(63)$ & $0.4193(55)$ & $0.4171(61)$ & $0.3929(64)$ \\
\hline 0.075 & $0.6401(63)$ & $0.5094(62)$ & $0.4692(54)$ & $0.4662(59)$ & $0.4453(65)$ \\
\hline \multirow[t]{2}{*}{0.1} & $0.7679(65)$ & $0.6401(61)$ & $0.6004(56)$ & $0.5966(61)$ & $0.5781(69)$ \\
\hline & \multicolumn{5}{|c|}{$\beta=5.65, M=2.1$ on $12^{3} \times 24$} \\
\hline 0.00 & $0.2418(68)$ & $0.1393(61)$ & $0.1067(60)$ & $0.0876(68)$ & $0.0839(67)$ \\
\hline 0.03 & $0.4143(70)$ & $0.3206(56)$ & $0.2903(55)$ & $0.2719(64)$ & $0.2689(64)$ \\
\hline 0.04 & $0.4722(74)$ & $0.3813(57)$ & $0.3521(55)$ & $0.3344(65)$ & $0.3327(67)$ \\
\hline 0.05 & $0.5295(77)$ & $0.4416(57)$ & $0.4134(55)$ & $0.3962(64)$ & $0.3955(69)$ \\
\hline 0.065 & $0.6175(82)$ & $0.5328(62)$ & $0.5056(60)$ & $0.4895(69)$ & $0.4888(77)$ \\
\hline 0.075 & $0.6740(83)$ & $0.5929(60)$ & $0.5666(58)$ & $0.5505(67)$ & $0.5505(76)$ \\
\hline \multirow[t]{2}{*}{0.1} & $0.8172(85)$ & $0.7439(58)$ & $0.7195(57)$ & $0.7037(68)$ & $0.7037(77)$ \\
\hline & \multicolumn{5}{|c|}{$\beta=5.65, M=2.5$ on $12^{3} \times 24$} \\
\hline 0.00 & $0.2624(63)$ & $0.1701(48)$ & $0.1546(43)$ & - & $0.1430(83)$ \\
\hline 0.03 & $0.4007(59)$ & $0.3049(49)$ & $0.2796(43)$ & - & $0.2439(77)$ \\
\hline 0.04 & $0.4496(59)$ & $0.3535(52)$ & $0.3270(46)$ & - & $0.2894(80)$ \\
\hline 0.05 & $0.4976(59)$ & $0.4012(56)$ & $0.3729(49)$ & - & $0.3320(83)$ \\
\hline 0.065 & $0.5682(61)$ & $0.4698(61)$ & $0.4385(54)$ & - & $0.3880(88)$ \\
\hline 0.075 & $0.6146(60)$ & $0.5153(63)$ & $0.4806(56)$ & - & $0.4236(89)$ \\
\hline 0.1 & $0.7284(60)$ & $0.6239(68)$ & $0.5783(60)$ & - & $0.4984(88)$ \\
\hline
\end{tabular}


TABLE XIII. Pion mass squared for the plaquette action in the strong coupling region at $\beta=5.65, M=1.3,1.7,2.1$ on $16^{3} \times 24$ lattice.

$\begin{array}{lllll}m_{f} & N_{s} & & \\ & & 20 & 30 & 40\end{array}$

$\beta=5.65, M=1.3$ on $16^{3} \times 24$

$\beta=5.65, M=1.7$ on $16^{3} \times 24$

$\begin{array}{llllll}0.00 & 0.2661(49) & 0.1358(56) & 0.0888(36) & 0.0599(38) & 0.0520(31) \\ 0.03 & 0.4179(51) & 0.2916(55) & 0.2434(34) & 0.2183(32) & 0.2086(30) \\ 0.04 & 0.4656(53) & 0.3411(57) & 0.2929(37) & 0.2693(33) & 0.2599(35) \\ 0.05 & 0.5137(55) & 0.3911(59) & 0.3426(40) & 0.3204(33) & 0.3113(38) \\ 0.065 & 0.5898(58) & 0.4687(65) & 0.4198(46) & 0.3992(37) & 0.3894(47) \\ 0.075 & 0.6392(59) & 0.5198(64) & 0.4707(48) & 0.4512(36) & 0.4414(48) \\ 0.1 & 0.7676(61) & 0.6513(66) & 0.6019(53) & 0.5849(36) & 0.5735(54)\end{array}$

$\beta=5.65, M=2.1$ on $16^{3} \times 24$

\begin{tabular}{|c|c|c|c|c|c|}
\hline 0.00 & $0.2317(40)$ & $0.1434(33)$ & $0.1132(72)$ & $0.0935(50)$ & $0.0815(38)$ \\
\hline 0.03 & $0.4052(46)$ & $0.3247(37)$ & $0.2973(62)$ & $0.2792(45)$ & $0.2675(43)$ \\
\hline 0.04 & $0.4629(50)$ & $0.3860(42)$ & $0.3604(63)$ & $0.3416(49)$ & $0.3317(47)$ \\
\hline 0.05 & $0.5202(55)$ & $0.4464(46)$ & $0.4226(62)$ & $0.4034(51)$ & $0.3945(51)$ \\
\hline 0.065 & $0.6085(62)$ & $0.5390(52)$ & $0.5166(68)$ & $0.4979(58)$ & $0.4902(60)$ \\
\hline 0.075 & $0.6652(65)$ & $0.5982(56)$ & $0.5774(63)$ & $0.5589(56)$ & $0.5509(61)$ \\
\hline 0.1 & $0.8095(72)$ & $0.7484(60)$ & $0.7299(60)$ & $0.7131(54)$ & $0.7036(66)$ \\
\hline
\end{tabular}


TABLE XIV. Pion mass squared for the RG improved action in the strong coupling region at $\beta=2.2, M=1.3,1.7,2.1,2.5$ on $12^{3} \times 24$ lattice.

\begin{tabular}{|c|c|c|c|c|c|}
\hline \multirow[t]{2}{*}{$m_{f}$} & \multicolumn{5}{|c|}{$N_{s}$} \\
\hline & \multicolumn{5}{|c|}{$\beta=2.2, M=1.3$ on $12^{3} \times 24$} \\
\hline 0.00 & $0.3038(55)$ & $0.1208(55)$ & $0.0684(71)$ & $0.0415(50)$ & $0.0389(60)$ \\
\hline 0.03 & $0.4002(52)$ & $0.2106(52)$ & $0.1579(64)$ & $0.1325(47)$ & $0.1253(57)$ \\
\hline 0.04 & $0.4301(52)$ & $0.2388(53)$ & $0.1867(63)$ & $0.1619(49)$ & $0.1518(59)$ \\
\hline 0.05 & $0.4604(52)$ & $0.2671(54)$ & $0.2154(61)$ & $0.1916(49)$ & $0.1786(60)$ \\
\hline 0.065 & $0.5083(52)$ & $0.3120(56)$ & $0.2605(64)$ & $0.2363(55)$ & $0.2215(64)$ \\
\hline 0.075 & $0.5395(52)$ & $0.3410(56)$ & $0.2894(61)$ & $0.2667(53)$ & $0.2494(63)$ \\
\hline 0.1 & $0.6209(51)$ & $0.4169(56)$ & $0.3647(58)$ & $0.3433(54)$ & $0.3228(60)$ \\
\hline
\end{tabular}

$\beta=2.2, M=1.7$ on $12^{3} \times 24$

\begin{tabular}{llllll}
\hline 0.00 & $0.1304(50)$ & $0.0575(50)$ & $0.0405(57)$ & $0.0408(42)$ & $0.0302(73)$ \\
0.03 & $0.2892(51)$ & $0.2186(46)$ & $0.2013(54)$ & $0.2049(39)$ & $0.1898(57)$ \\
0.04 & $0.3394(54)$ & $0.2697(48)$ & $0.2529(56)$ & $0.2588(40)$ & $0.2410(55)$ \\
0.05 & $0.3900(57)$ & $0.3210(49)$ & $0.3042(58)$ & $0.3119(40)$ & $0.2917(51)$ \\
0.065 & $0.4695(64)$ & $0.4016(55)$ & $0.3850(66)$ & $0.3961(48)$ & $0.3724(56)$ \\
0.075 & $0.5213(64)$ & $0.4540(54)$ & $0.4372(68)$ & $0.4486(46)$ & $0.4234(53)$ \\
0.1 & $0.6559(67)$ & $0.5904(56)$ & $0.5737(77)$ & $0.5863(47)$ & $0.5582(58)$ \\
\hline
\end{tabular}

$\beta=2.2, M=2.1$ on $12^{3} \times 24$

\begin{tabular}{llllll}
\hline 0.00 & $0.1159(64)$ & $0.0810(63)$ & $0.0645(68)$ & $0.0556(73)$ & $0.0415(82)$ \\
0.03 & $0.2879(60)$ & $0.2510(55)$ & $0.2303(64)$ & $0.2272(71)$ & $0.2131(73)$ \\
0.04 & $0.3461(63)$ & $0.3083(56)$ & $0.2873(66)$ & $0.2848(75)$ & $0.2705(74)$ \\
0.05 & $0.4038(65)$ & $0.3644(58)$ & $0.3429(68)$ & $0.3415(78)$ & $0.3274(72)$ \\
0.065 & $0.4911(72)$ & $0.4519(64)$ & $0.4276(76)$ & $0.4292(87)$ & $0.4143(81)$ \\
0.075 & $0.5480(70)$ & $0.5068(66)$ & $0.4817(73)$ & $0.4851(85)$ & $0.4706(76)$ \\
0.1 & $0.6906(72)$ & $0.6482(71)$ & $0.6197(69)$ & $0.6279(84)$ & $0.6136(76)$ \\
\hline
\end{tabular}

$\beta=2.2, M=2.5$ on $12^{3} \times 24$

\begin{tabular}{llllll}
\hline 0.00 & $0.1549(48)$ & $0.1020(60)$ & $0.1127(63)$ & - & - \\
0.03 & $0.2814(47)$ & $0.2208(57)$ & $0.2184(53)$ & - & - \\
0.04 & $0.3256(48)$ & $0.2649(57)$ & $0.2611(54)$ & - & - \\
0.05 & $0.3692(51)$ & $0.3076(58)$ & $0.3018(53)$ & - & - \\
0.065 & $0.4329(56)$ & $0.3699(59)$ & $0.3607(54)$ & - & - \\
0.075 & $0.4754(59)$ & $0.4099(59)$ & $0.3965(52)$ & - & - \\
\hline .1 & $0.5793(65)$ & $0.5058(58)$ & $0.4797(48)$ & - \\
\hline
\end{tabular}


TABLE XV. Pion mass squared for the RG improved action in the strong coupling region at $\beta=2.2, M=1.3,1.7,2.1$ on $16^{3} \times 24$ lattice.

\begin{tabular}{|c|c|c|c|c|c|}
\hline$m_{f}$ & 10 & 20 & $\begin{array}{l}N_{s} \\
30\end{array}$ & 40 & 50 \\
\hline & \multicolumn{5}{|c|}{$\beta=2.2, M=1.3$ on $16^{3} \times 24$} \\
\hline 0.00 & $0.3126(54)$ & - & $0.0709(19)$ & - & $0.0464(30)$ \\
\hline 0.03 & $0.4095(56)$ & - & $0.1609(19)$ & - & $0.1347(28)$ \\
\hline 0.04 & $0.4400(58)$ & - & $0.1900(19)$ & - & $0.1634(29)$ \\
\hline 0.05 & $0.4707(59)$ & - & $0.2190(21)$ & - & $0.1918(30)$ \\
\hline 0.065 & $0.5194(62)$ & - & $0.2642(25)$ & - & $0.2368(34)$ \\
\hline 0.075 & $0.5508(63)$ & - & $0.2938(26)$ & - & $0.2652(34)$ \\
\hline \multirow[t]{2}{*}{0.1} & $0.6326(66)$ & - & $0.3702(33)$ & - & $0.3395(35)$ \\
\hline & \multicolumn{5}{|c|}{$\beta=2.2, M=1.7$ on $16^{3} \times 24$} \\
\hline 0.00 & $0.1276(49)$ & $0.0579(37)$ & $0.0392(36)$ & $0.0308(35)$ & $0.0246(35)$ \\
\hline 0.03 & $0.2882(46)$ & $0.2222(34)$ & $0.2012(32)$ & $0.1959(33)$ & $0.1908(34)$ \\
\hline 0.04 & $0.3392(45)$ & $0.2763(36)$ & $0.2527(35)$ & $0.2498(37)$ & $0.2454(36)$ \\
\hline 0.05 & $0.3905(44)$ & $0.3300(38)$ & $0.3042(37)$ & $0.3034(40)$ & $0.2995(38)$ \\
\hline 0.065 & $0.4704(46)$ & $0.4131(44)$ & $0.3854(42)$ & $0.3874(46)$ & $0.3837(44)$ \\
\hline 0.075 & $0.5228(45)$ & $0.4667(45)$ & $0.4381(42)$ & $0.4409(48)$ & $0.4378(45)$ \\
\hline \multirow[t]{2}{*}{0.1} & $0.6582(45)$ & $0.6046(48)$ & $0.5760(47)$ & $0.5798(53)$ & $0.5773(47)$ \\
\hline & \multicolumn{5}{|c|}{$\beta=2.2, M=2.1$ on $16^{3} \times 24$} \\
\hline 0.00 & $0.1194(51)$ & $0.0735(40)$ & $0.0539(46)$ & $0.0455(41)$ & $0.0492(46)$ \\
\hline 0.03 & $0.2868(42)$ & $0.2442(38)$ & $0.2303(45)$ & $0.2177(40)$ & $0.2238(44)$ \\
\hline 0.04 & $0.3425(40)$ & $0.3020(40)$ & $0.2912(49)$ & $0.2754(43)$ & $0.2840(48)$ \\
\hline 0.05 & $0.3980(40)$ & $0.3590(41)$ & $0.3509(51)$ & $0.3324(45)$ & $0.3427(49)$ \\
\hline 0.065 & $0.4826(43)$ & $0.4459(47)$ & $0.4413(59)$ & $0.4202(53)$ & $0.4336(58)$ \\
\hline 0.075 & $0.5376(43)$ & $0.5019(48)$ & $0.4991(58)$ & $0.4763(51)$ & $0.4899(56)$ \\
\hline 0.1 & $0.6770(49)$ & $0.6434(54)$ & $0.6445(59)$ & $0.6197(51)$ & $0.6336(56)$ \\
\hline
\end{tabular}

TABLE XVI. Pion mass squared for the plaquette action in the weak coupling region at $\beta=6.0, M=1.8$ on $16^{3} \times 32$ lattice.

\begin{tabular}{|c|c|c|c|c|c|}
\hline \multirow[t]{2}{*}{$m_{f}$} & \multicolumn{5}{|c|}{$N_{s}$} \\
\hline & 4 & 10 & 16 & 20 & 30 \\
\hline 0.0 & $0.0891(49)$ & $0.0096(36)$ & $0.0025(27)$ & $0.0063(21)$ & $0.0019(20)$ \\
\hline 0.02 & $0.1617(43)$ & $0.0795(30)$ & $0.0711(24)$ & $0.0736(19)$ & $0.0703(17)$ \\
\hline 0.03 & $0.1959(41)$ & $0.1130(34)$ & $0.1032(25)$ & $0.1051(20)$ & $0.1029(21)$ \\
\hline 0.04 & $0.2310(39)$ & $0.1471(32)$ & $0.1359(24)$ & $0.1371(20)$ & $0.1362(20)$ \\
\hline 0.04 & $0.2311(40)$ & $0.1471(36)$ & $0.1362(26)$ & $0.1380(21)$ & $0.1366(23)$ \\
\hline 0.05 & $0.2671(38)$ & $0.1823(35)$ & $0.1710(27)$ & $0.1714(23)$ & $0.1706(20)$ \\
\hline 0.06 & $0.3041(36)$ & $0.2179(34)$ & $0.2059(27)$ & $0.2061(23)$ & $0.2059(20)$ \\
\hline
\end{tabular}

TABLE XVII. Pion mass squared for the RG improved action in the weak coupling region at $\beta=2.6, M=1.8$ on $16^{3} \times 32$ lattice.

\begin{tabular}{|c|c|c|c|c|c|}
\hline \multirow[t]{2}{*}{$m_{f}$} & \multicolumn{5}{|c|}{$N_{s}$} \\
\hline & 4 & 10 & 16 & 20 & 24 \\
\hline 0.0 & $0.0536(26)$ & $0.0044(33)$ & $0.0052(23)$ & $0.0023(19)$ & $0.0022(16)$ \\
\hline 0.02 & $0.1188(24)$ & $0.0670(30)$ & $0.0671(23)$ & $0.0646(15)$ & $0.0652(14)$ \\
\hline 0.03 & $0.1505(26)$ & $0.0967(32)$ & $0.0969(26)$ & $0.0942(17)$ & $0.0959(15)$ \\
\hline 0.04 & $0.1826(29)$ & $0.1268(30)$ & $0.1270(27)$ & $0.1243(17)$ & $0.1268(15)$ \\
\hline 0.04 & $0.1835(29)$ & $0.1274(34)$ & $0.1280(29)$ & $0.1246(19)$ & $0.1274(16)$ \\
\hline 0.05 & $0.2150(36)$ & $0.1583(31)$ & $0.1582(31)$ & $0.1559(18)$ & $0.1580(16)$ \\
\hline 0.06 & $0.2491(39)$ & $0.1907(33)$ & $0.1904(31)$ & $0.1877(18)$ & $0.1908(16)$ \\
\hline
\end{tabular}


[1] D. Kaplan, Phys. Lett. B 288, 342 (1992); Nucl. Phys. B (Proc. Suppl.) 30, 597 (1993).

[2] M. Golterman, K. Jansen, and D. Kaplan, Phys. Lett. B 301, 219 (1993).

[3] R. Narayanan and H. Neuberger, Nucl. Phys. B443, 305 (1995).

[4] Y. Shamir, Nucl. Phys. B406, 90 (1993).

[5] V. Furman and Y. Shamir, Nucl. Phys. B439, 54 (1995).

[6] H. Neuberger, Phys. Lett. B 417, 141 (1998); 427, 353 (1998).

[7] P. Hasenfratz, V. Laliena, and F. Niedermayer, Phys. Lett. B 427, 125 (1998).

[8] M. Lüscher, Phys. Lett. B 428, 342 (1998).

[9] P. Ginsparg and K. Wilson, Phys. Rev. D 25, 2649 (1982).

[10] H. Neuberger, Phys. Rev. D 57, 5417 (1998).

[11] Y. Kikukawa and T. Noguchi, hep-lat/9902022.

[12] S. Aoki and Y. Taniguchi, Phys. Rev. D 59, 054510 (1999).

[13] Y. Kikukawa, H. Neuberger, and A. Yamada, Nucl. Phys. B526, 572 (1998).

[14] S. Aoki, T. Izubuchi, Y. Kuramashi, and Y. Taniguchi, Phys. Rev. D 59, 094505 (1999).

[15] T. Blum and A. Soni, Phys. Rev. D 56, 174 (1997); Phys. Rev. Lett. 79, 3595 (1997); hep-lat/9712004.

[16] For a review, see T. Blum, Nucl. Phys. B (Proc. Suppl.) 73, 167 (1999), and references therein.

[17] J. F. Lagae and D. K. Sinclair, Nucl. Phys. B (Proc. Suppl.) 83-84, 405 (2000).

[18] T. Blum, A. Soni, and M. Wingate, Nucl. Phys. B (Proc. Suppl.) 73, 201 (1999); Phys. Rev. D 60, 114507 (1999).

[19] M. Wingate et al., Nucl. Phys. B (Proc. Suppl.) 83-84, 221 (2000).

[20] C. Dawson et al., Nucl. Phys. B (Proc. Suppl.) 83-84, 854 (2000)

[21] S. Aoki, T. Izubuchi, Y. Kuramashi, and Y. Taniguchi, Nucl. Phys. B (Proc. Suppl.) 83-84, 624 (2000); Phys. Rev. D 62 , 094003 (2000).

[22] S. Sasaki et al., Nucl. Phys. B (Proc. Suppl.) 83-84, 206 (2000).

[23] L. Wu et al., Nucl. Phys. B (Proc. Suppl.) 83-84, 224 (2000).

[24] P. Chen, N. Christ, G. Fleming, A. Kaehler, C. Malureanu, R. Mawhinney, G. Siegert, C. Sui, P. Vranas, and Y. Zhestkov, Nucl. Phys. B (Proc. Suppl.) 73, 456 (1999).

[25] P. Vranas et al., hep-lat/9903024.
[26] P. Vranas et al., Nucl. Phys. B (Proc. Suppl.) 83-84, 414 (2000).

[27] For a review, see P. Chen, N. Christ, G. Fleming, A. Kaehler, C. Malureanu, R. Mawhinney, G. Siegert, C. Sui, P. M. Vranas, and Y. Zhestkov, hep-lat/9812011, and references therein.

[28] G. T. Fleming et al., Nucl. Phys. B (Proc. Suppl.) 83-84, 363 (2000).

[29] CP-PACS Collaboration, A. Ali Khan et al., Nucl. Phys. B (Proc. Suppl.) 83-84, 591 (2000).

[30] Y. Iwasaki, UTHEP-118, 1983.

[31] R. G. Edwards, U. M. Heller, and T. R. Klassen, Nucl. Phys. B517, 377 (1998).

[32] Y. Iwasaki, K. Kanaya, T. Kaneko, and T. Yoshie, Phys. Rev. D 56, 151 (1997).

[33] CP-PACS Collaboration, M. Okamoto et al., Phys. Rev. D 60, 094510 (1999).

[34] CP-PACS Collaboration, A. Ali Khan et al., Nucl. Phys. B (Proc. Suppl.) 83-84, 176 (2000).

[35] P. Hernandez, K. Jansen, and M. Lüscher, Nucl. Phys. B552, 363 (1999).

[36] Y. Kikukawa, Nucl. Phys. B584, 511 (2000).

[37] S. Aoki, Phys. Rev. D 30, 2653 (1984); Phys. Rev. Lett. 57, 3136 (1986); Nucl. Phys. B314, 79 (1989).

[38] R. Setoodeh, C. T. H. Davies, and I. M. Barbour, Phys. Lett. B 213, 195 (1988)

[39] S. Aoki and A. Gocksch, Phys. Lett. B 231, 449 (1989).

[40] S. Aoki, T. Kaneda, and A. Ukawa, Phys. Rev. D 56, 1808 (1997).

[41] S. Sharpe and R. L. Singleton, Jr., Phys. Rev. D 58, 074501 (1998).

[42] R. G. Edwards, U. M. Heller, and R. Narayanan, Phys. Rev. D 60, 034502 (1999); for a review, see hep-lat/0001013, and references therein.

[43] Y. Iwasaki and T. Yoshie, Phys. Lett. 125B, 197 (1983); 131B, 159 (1983); S. Itoh, Y. Iwasaki, and T. Yoshie, ibid. 147B, 141 (1984).

[44] CP-PACS Collaboration (in preparation).

[45] JLQCD Collaboration, S. Aoki et al., Phys. Rev. Lett. 80, 5271 (1998); and (unpublished).

[46] R. Gupta, G. Guralnik, G. W. Kilcup, and S. R. Sharpe, Phys. Rev. D 43, 2003 (1991).

[47] S. Aoki, T. Umemura, M. Fukugita, N. Ishizuka, H. Mino, M. Okawa, and A. Ukawa, Phys. Rev. D 50, 486 (1994). 